\title{
Killing Tensors and Symmetries
}

\author{
David Garfinkle \\ Physics Department, Oakland University, Rochester, MI \\ E.N. Glass \\ Physics Department, University of Michigan, Ann Arbor, MI
}

(Dated: March 11, 2010)

\begin{abstract}
A new method is presented for finding Killing tensors in spacetimes with symmetries. The method is used to find all the Killing tensors of Melvin's magnetic universe and the Schwarzschild vacuum. We show that they are all trivial. The method requires less computation than solving the full Killing tensor equations directly, and it can be used even when the spacetime is not algebraically special

PACS numbers: 04.20.Cv, 04.20.Jb
\end{abstract}

\section{INTRODUCTION}

Killing tensors are useful because, like Killing vectors, they provide conserved quantities for geodesic motion, most famously in the Kerr metric where the Killing tensor gives rise to the Carter constant [1]. However, it is much more difficult to find Killing tensors than Killing vectors. In 4 spacetime dimensions, the equation for a Killing tensor becomes 20 partial differential equations for 10 functions of 4 variables. Most of the known results on Killing tensors come from the fact that the Killing tensor equation simplifies in certain classes of algebraically special spacetimes [2, 3]. However, equations in general relativity also often simplify in the presence of symmetry [4]. We will show that the Killing tensor equation simplifies when a spacetime possesses a hypersurface orthogonal Killing vector, and that this simplification provides an effective method for finding Killing tensors. We will apply this method to Melvin's magnetic universe [5] and the Schwarzschild vacuum solution. It is shown that all of their Killing tensors are trivial in the sense that they are either the metric or the symmetrized product of Killing vectors. The method is presented in section 2. It is applied to Melvin's magnetic universe in section 3 and the Schwarzschild vacuum in section 4. Conclusions are given in section 5 . 
Notation: Lower case Latin indices, $B^{a}$, range over n-dimensions. Greek indices, $B^{\mu}$, range over n-1 dimensions. For Killing vector $\xi^{a}$ an overdot will denote a Lie derivative, $\dot{A}:=\mathcal{L}_{\xi} A$.

\section{THE KILLING TENSOR METHOD}

A Killing tensor (of order 2) is a symmetric tensor $X_{a b}$ that satisfies

$$
\nabla_{(a} X_{b c)}=0
$$

Now suppose that the spacetime has a hypersurface orthogonal Killing vector $\xi^{a}$. Define $V$ such that

$$
\xi^{a} \xi_{a}=\epsilon V^{2}
$$

where $\epsilon= \pm 1$. Then the metric in directions orthogonal to $\xi^{a}$ is given by

$$
h_{a b}=g_{a b}-\epsilon V^{-2} \xi_{a} \xi_{b}
$$

One can use $h_{b}^{a}$ as a projection operator to project any tensor in directions orthogonal to $\xi^{a}$. In particular, the Killing tensor can be decomposed as

$$
X_{a b}=A \xi_{a} \xi_{b}+2 B_{(a} \xi_{b)}+C_{a b}
$$

where $B_{a}$ and $C_{a b}$ are orthogonal to $\xi^{a}$. The Killing tensor equation for this decomposition is

$$
\nabla_{(a} A \xi_{b} \xi_{c)}+2 \nabla_{(a} B_{b} \xi_{c)}+\nabla_{(a} C_{b c)}=0 .
$$

Projecting the Killing tensor equation using all combinations of $h^{a}{ }_{b}$ and $\xi^{a}$ yields the following

$$
\begin{aligned}
D_{(a} C_{b c)} & =0, \\
\mathcal{L}_{\xi} A & =-2 V^{-1} B^{a} D_{a} V, \\
\mathcal{L}_{\xi} B_{a} & =-V^{-1} C_{a b} D^{b} V-\frac{1}{2} \epsilon V^{2} D_{a} A, \\
\mathcal{L}_{\xi} C_{a b} & =-2 \epsilon V^{2} D_{(a} B_{b)} .
\end{aligned}
$$

Here $D_{a}$ is the derivative operator associated with the metric $h_{a b}$ and $\mathcal{L}_{\xi}$ denotes the Lie derivative with respect to Killing vector $\xi^{a}$. Solving Eq.(8) for $D_{a} A$ yields

$$
D_{a} A=-(2 / \epsilon)\left[V^{-2} \mathcal{L}_{\xi} B_{a}+V^{-3} C_{a b} D^{b} V\right]
$$


The left hand side of Eq.(10) is curl-free and thus the curl of the right hand side must vanish. Hence

$$
D_{[a}\left(V^{-2} \mathcal{L}_{\xi} B_{b]}\right)+D_{[a}\left(V^{-3} C_{b] c} D^{c} V\right)=0
$$

Furthermore, since $\mathcal{L}_{\xi}$ commutes with $D_{a}$ it follows that $\mathcal{L}_{\xi}$ of the right hand side of Eq.(10) equals $D_{a}$ of the right hand side of Eq.(7), and therefore

$$
\mathcal{L}_{\xi} \mathcal{L}_{\xi} B_{a}+V^{-1}\left(D^{b} V\right) \mathcal{L}_{\xi} C_{a b}-\epsilon V^{2} D_{a}\left(V^{-1} B^{b} D_{b} V\right)=0 .
$$

Equations (11) and (12) provide the integrability conditions for equations (71) and (10).

These equations are most easily implemented in a coordinate system adapted to the Killing vector. Choose a coordinate system $\left(y, x^{\mu}\right)$ such that $x^{\mu}$ are coordinates on the surface orthogonal to the Killing vector and $\mathcal{L}_{\xi}$ is simply a partial derivative with respect to $y$. Use $\partial_{\mu}$ or a comma to denote a derivative with respect to the $x^{\mu}$ coordinates. The Latin indices in this section are n-dimensional, and the method below projects objects and equations down to n-1 dimensions with Greek indices.

Equation (91) becomes

$$
\dot{C}_{\mu \nu}=-\epsilon V^{2}\left(B^{\alpha} \partial_{\alpha} h_{\mu \nu}+h_{\alpha \nu} \partial_{\mu} B^{\alpha}+h_{\mu \alpha} \partial_{\nu} B^{\alpha}\right)
$$

while integrability conditions (11) and (12) become

$$
\begin{aligned}
\partial_{[\mu}\left(V^{-2} \dot{B}_{\nu]}\right)+\partial_{[\mu}\left(V^{-3} C_{\nu] \alpha} \partial^{\alpha} V\right) & =0 \\
\ddot{B}_{\mu}+V^{-1} \dot{C}_{\mu \nu} \partial^{\nu} V-\epsilon V^{2} \partial_{\mu}\left(V^{-1} B^{\nu} \partial_{\nu} V\right) & =0 .
\end{aligned}
$$

Equations (7) and (10) for $A$ are written as

$$
\begin{aligned}
\dot{A} & =-2 V^{-1} B^{\mu} \partial_{\mu} V \\
\partial_{\mu} A & =-(2 / \epsilon)\left(V^{-2} \dot{B}_{\mu}+V^{-3} C_{\mu \nu} \partial^{\nu} V\right)
\end{aligned}
$$

Note that equations (13-17) can be evaluated without ever having to calculate a Christoffel symbol.

The complete set of Killing tensors can be found as follows: first solve Eq.(66) for the most general $C_{\mu \nu}$. Note that this general solution will contain arbitrary "constants" that are really functions of the Killing coordinate. Now using the general $C_{\mu \nu}$, find the most general $B_{\mu}$ satisfying Eq.(13). Then restrict this general solution by demanding that equations (14) and (15) also be satisfied. Finally, solve equations (16) and (17) for $A$. 


\section{KILLING TENSORS OF THE MELVIN METRIC}

Melvin's magnetic universe is a static, cylindrically symmetric, Petrov type D solution of the Einstein-Maxwell equations. Its metric is usually written as

$$
d \tilde{s}_{\mathrm{Mel}}^{2}=a^{2}\left(-d \tilde{t}^{2}+d \rho^{2}+d \tilde{z}^{2}\right)+\left(\rho^{2} / a^{2}\right) d \tilde{\phi}^{2}
$$

where the function $a$ is

$$
a=1+\frac{1}{4} B_{0}^{2} \rho^{2}
$$

The constant $B_{0}$ is the value of the magnetic field on the $\rho=0$ axis. Define coordinates $t=\left(B_{0} / 2\right) \tilde{t}, r=\left(B_{0} / 2\right) \rho, z=\left(B_{0} / 2\right) \tilde{z}, \phi=\tilde{\phi}$. In terms of these coordinates we have

$$
a=1+r^{2}
$$

while the metric becomes $d \tilde{s}_{\text {Mel }}^{2}=\left(4 / B_{0}^{2}\right) d s_{\text {Mel }}^{2}$ with

$$
d s_{\text {Mel }}^{2}=a^{2}\left(-d t^{2}+d r^{2}+d z^{2}\right)+\left(r^{2} / a^{2}\right) d \phi^{2} .
$$

Since $d \tilde{s}_{\text {Mel }}^{2}$ and $d s_{\text {Mel }}^{2}$ differ only by an overall constant scale, they have the same Killing

vectors and Killing tensors. For simplicity, we will work with metric $d s_{\mathrm{Mel}}^{2}$. Since the metric components are independent of $t, \phi$, and $z$, it follows that there are Killing vectors for each of these coordinate directions. They are denoted by $(t, \phi, z) \rightarrow\left(\tau^{\mu}, \eta^{\mu}, \lambda^{\mu}\right)$ respectively. Each of these Killing vectors is hypersurface orthogonal. In addition, the metric has boost symmetry in the $t z$ plane, with corresponding Killing field $t \lambda^{a}+z \tau^{a}$.

We will use the method of the previous section to work out the Killing tensors of Melvin's magnetic universe. First we will find the Killing tensors of the 2-dimensional $r z$ surface, then use these to find the Killing tensors of the 3-dimensional $r z \phi$ surface, and finally find the Killing tensors of the 4-dimensional Melvin metric.

We will use $c_{1}, c_{2}$ etc. to denote constants, and $k_{1}, k_{2}$ etc. to denote quantities that depend only on the coordinate associated with the Killing vector.

\section{A. An $r z$ surface}

The $r z 2$-surface has a metric of form

$$
g_{a b} d x^{a} d x^{b}=a^{2}\left(d r^{2}+d z^{2}\right)
$$


We have a $z$ coordinate Killing vector $\lambda^{\mu}$ for which $V=a$ and $\epsilon=1$, and a 1-dimensional metric $h_{\mu \nu}=a^{2} r_{, \mu} r_{, \nu}$ orthogonal to the Killing vector. Since the r-direction is a 1dimensional line, it follows that the Killing tensor must take the form $C_{\mu \nu}=F h_{\mu \nu}$ for some scalar $F$. It then follows from Eq.(6) that $F$ is independent of $r$. We therefore have

$$
C_{\mu \nu}=k_{1} h_{\mu \nu}=k_{1} a^{2} r_{, \mu} r_{, \nu}
$$

for some $k_{1}(z)$. Equation (13) then becomes, with $B^{\mu} \rightarrow B^{r}$ only

$$
\dot{C}_{r r}=\dot{k}_{1} a^{2}=-a^{2}\left(\frac{d a^{2}}{d r} B^{r}+2 a^{2} \partial_{r} B^{r}\right)
$$

or

$$
\dot{k}_{1}=-2 a^{2}\left(\frac{d B^{r}}{d r}+\frac{1}{a} \frac{d a}{d r} B^{r}\right)
$$

The general solution for $B^{r}$ is

$$
B^{r}=a^{-1}\left(k_{2}-\frac{\dot{k}_{1}}{2} \arctan r\right)
$$

Equation (14) is automatically satisfied, while Eq.(15) becomes

$$
0=\left(-\frac{1}{2} \dddot{k}_{1}-2 \dot{k}_{1}\right) a^{2} \arctan r+3 \dot{k}_{1} a \arctan r+\left(\ddot{k}_{2}+4 k_{2}\right) a^{2}+3 \dot{k}_{1} r-6 k_{2} a
$$

Here we have grouped terms so that each term is a coefficient independent of $r$ multiplied by a function of $r$ and the functions of $r$ are linearly independent. Thus each coefficient must vanish, which yields $k_{2}=0$ and $\dot{k}_{1}=0$, from which it follows that $k_{1}=c_{1}$ and $B^{r}=0$. It then follows from Eq.(77) that $A$ is independent of $z$. From Eq.(17) it follows that

$$
A(r)=c_{2}+c_{1} a^{-2}
$$

Using Eq.(4) we find that the general Killing tensor of the $r z$ surface is

$$
X_{a b}=c_{1} g_{a b}+c_{2} \lambda_{a} \lambda_{b}
$$

\section{B. An $r z \phi$ surface}

We now consider an $r z \phi$ surface with metric

$$
g_{a b} d x^{a} d x^{b}=a^{2}\left(d r^{2}+d z^{2}\right)+\left(r^{2} / a^{2}\right) d \phi^{2} .
$$


The Killing field is $\eta^{a}$, with $\epsilon=1$ and $V=r / a$. Here $h_{\mu \nu}$ is the $g_{a b}$ of the previous subsection, while $C_{\mu \nu}$ is the $X_{a b}$ of the previous subsection. We have

$$
\begin{aligned}
& h_{\mu \nu}=a^{2}\left(r,_{\mu} r,_{\nu}+z,,_{\mu} z,_{\nu}\right) \\
& C_{\mu \nu}=k_{1} h_{\mu \nu}+k_{2} \lambda_{\mu} \lambda_{\nu} .
\end{aligned}
$$

With $B^{\mu} \rightarrow\left(B^{r}, B^{z}\right)$, the $r r, z z$, and $r z$ components of Eq.(13) are then, respectively

$$
\begin{aligned}
\dot{k}_{1} a^{2} & =-2 \frac{r^{2}}{a^{2}}\left(a \frac{d a}{d r} B^{r}+a^{2} \partial_{r} B^{r}\right) \\
\dot{k}_{1} a^{2}+\dot{k}_{2} a^{4} & =-2 \frac{r^{2}}{a^{2}}\left(a \frac{d a}{d r} B^{r}+a^{2} \partial_{z} B^{z}\right) \\
0 & =\partial_{r} B^{z}+\partial_{z} B^{r}
\end{aligned}
$$

From Eq.(33) we find that $B^{r}$ must take the form

$$
B^{r}=\frac{1}{a}\left[F(z, \phi)-\frac{\dot{k}_{1}}{2}\left(-\frac{1}{r}+3 r+r^{3}+\frac{r^{5}}{5}\right)\right]
$$

for some function $F(z, \phi)$. Then using Eq.(36) and integrating Eq.(35) we find

$$
B^{z}=G(z, \phi)-\left(\frac{\partial F}{\partial z}\right) \arctan r
$$

for some function $G(z, \phi)$. Finally, substituting the expressions in equations (36) and (37) into Eq. (34) we find

$$
-\frac{1}{2 r^{2}}\left(\dot{k}_{1} a^{2}+\dot{k}_{2} a^{4}\right)=\frac{\partial G}{\partial z}-\left(\frac{\partial^{2} F}{\partial z^{2}}\right) \arctan r+2 F\left(\frac{r}{a^{2}}\right)-\frac{\dot{k}_{1}}{a^{2}}\left(-1+3 r^{2}+r^{4}+\frac{r^{6}}{5}\right)
$$

The only odd functions of $r$ in this equation are arctan $r$ and $r / a^{2}$ and these functions are linearly independent, so the coefficient of each must vanish. This implies that $F=0$. Furthermore, in order that the left hand side not diverge as $r \rightarrow 0$ we must have $\dot{k}_{2}=-\dot{k}_{1}$. Equation (38) then becomes

$$
\frac{\partial G}{\partial z}=\dot{k}_{1}\left[\frac{a^{2}(1+a)}{2}+\frac{1}{a^{2}}\left(-1+3 r^{2}+r^{4}+\frac{r^{6}}{5}\right)\right]
$$

It then follows that both $\dot{k}_{1}$ and $\partial G / \partial z$ must vanish. Thus, we have found that $C_{\mu \nu}$ and $B^{\mu}$ take the form

$$
\begin{aligned}
C_{\mu \nu} & =c_{1} h_{\mu \nu}+c_{2} \lambda_{\mu} \lambda_{\nu} \\
B^{\mu} & =k_{4}(\phi) \lambda^{\mu}
\end{aligned}
$$


for some function $k_{4}(\phi)$. We now impose the integrability condition Eq.(14) which forces $\dot{k}_{4}$ to vanish. This implies $k_{4}=c_{4}$ and thus $B^{\mu}=c_{4} \lambda^{\mu}$. It then follows from Eq.(16) that $A$ is independent of $\phi$. Equation (17) then becomes

$$
\partial_{\mu} A=c_{1} \partial_{\mu}(r / a)^{-2}
$$

for which the solution is

$$
A(r)=c_{3}+c_{1} \frac{a^{2}}{r^{2}}
$$

Thus the general Killing tensor of the $r z \phi$ surface is

$$
X_{a b}=c_{1} g_{a b}+c_{2} \lambda_{a} \lambda_{b}+c_{3} \eta_{a} \eta_{b}+2 c_{4} \lambda_{(a} \eta_{b)} .
$$

\section{The Melvin metric}

We are now ready to treat the full Melvin metric by adding the $\tau^{a}$ Killing field to the metric of the previous subsection. We have

$$
\begin{gathered}
h_{\mu \nu}=a^{2}\left(r_{\mu} r_{, \nu}+z,_{\mu} z,_{\nu}\right)+\left(r^{2} / a^{2}\right) \phi_{, \mu} \phi_{,_{\nu}} \\
C_{\mu \nu}=k_{1} h_{\mu \nu}+k_{2} \lambda_{\mu} \lambda_{\nu}+k_{3} \eta_{\mu} \eta_{\nu}+2 k_{4} \lambda_{(\mu} \eta_{\nu)}
\end{gathered}
$$

The $\tau^{a}$ Killing vector has $\epsilon=-1$ and $V=a$. Equation (13) for $\dot{C}_{\mu \nu}$ becomes the following:

$$
\begin{aligned}
\dot{k}_{1} & =2 a^{2} \partial_{r} B^{r}+2 a \frac{d a}{d r} B^{r} \\
\dot{k}_{1}+\dot{k}_{2} a^{2} & =2 a \frac{d a}{d r} B^{r}+2 a^{2} \partial_{z} B^{z} \\
a^{-2} \dot{k}_{1}+\frac{r^{2}}{a^{4}} \dot{k}_{3} & =2 \partial_{\phi} B^{\phi}+2\left(\frac{1}{r}-\frac{1}{a} \frac{d a}{d r}\right) B^{r} \\
0 & =\partial_{r} B^{z}+\partial_{z} B^{r} \\
0 & =a^{2} \partial_{\phi} B^{r}+\frac{r^{2}}{a^{2}} \partial_{r} B^{\phi} \\
\dot{k}_{4} & =\frac{a^{4}}{r^{2}} \partial_{\phi} B^{z}+\partial_{z} B^{\phi}
\end{aligned}
$$

Solving Eq.(47) we find

$$
B^{r}=\frac{1}{a}\left[\frac{\dot{k}_{1}}{2} \arctan r+F(z, \phi, t)\right]
$$

for some function $F(z, \phi, t)$. Now, using this result in Eq.(50) we find

$$
B^{z}=-\frac{\partial F}{\partial z} \arctan r+G(z, \phi, t)
$$


for some function $G(z, \phi, t)$. Using equations (53) and (54) in Eq.(48), we obtain

$$
0=-\dot{k}_{1}+\left(2 \frac{\partial G}{\partial z}-\dot{k}_{2}\right) a^{2}+4 F r+2 \dot{k}_{1} r \arctan r-2\left(\frac{\partial^{2} F}{\partial z^{2}}\right) a^{2} \arctan r
$$

Here we have grouped terms so that each term consists of a function of $r$ multiplied by a coefficient that is independent of $r$. Since the functions of $r$ are linearly independent, it follows that each coefficient vanishes. We then find that $F=0$, and that $k_{1}=c_{1}$, and that $G=\dot{k}_{2} z / 2+h(\phi, t)$ for some function $h(\phi, t)$. That is, $B^{r}$ vanishes, and $B^{z}$ takes the form

$$
B^{z}=\frac{1}{2} \dot{k}_{2} z+h(\phi, t)
$$

Equations (49,51,52) then become

$$
\begin{aligned}
\left(\frac{r^{2}}{a^{4}}\right) \dot{k}_{3} & =2 \partial_{\phi} B^{\phi} \\
0 & =\partial_{r} B^{\phi} \\
\dot{k}_{4} & =\left(\frac{a^{4}}{r^{2}}\right) \frac{\partial h}{\partial \phi}+\partial_{z} B^{\phi}
\end{aligned}
$$

Differentiating equations (57) and (59) with respect to $r$, and using Eq.(58), it follows that $\dot{k}_{3}=0$ and $\partial h / \partial \phi=0$. Thus we have $k_{3}=c_{3}$ for constant $c_{3}$, and $h=k_{5}$ for some function $k_{5}(t)$, and $B^{\phi}=\dot{k}_{4} z+k_{6}$ for function $k_{6}(t)$. $B^{\mu}$ takes the form

$$
B^{\mu}=\left(\frac{1}{2} \dot{k}_{2} z+k_{5}\right) \lambda^{\mu}+\left(\dot{k}_{4} z+k_{6}\right) \eta^{\mu}
$$

We therefore have

$$
a^{-2} \dot{B}_{\mu}=\left(\frac{1}{2} \ddot{k}_{2} z+\dot{k}_{5}\right) \partial_{\mu} z+\frac{r^{2}}{a^{4}}\left(\ddot{k}_{4} z+\dot{k}_{6}\right) \partial_{\mu} \phi
$$

Using the expression in Eq.([61), we find that Eq.(14) becomes

$$
0=\ddot{k}_{4} z+\dot{k}_{6}
$$

From Eq.(62) we find that $k_{6}=c_{6}$ and $k_{4}=c_{4} t+c_{7}$ for constants $c_{4}, c_{6}$, and $c_{7}$. Using the expression for $B^{\mu}$ of Eq.(60) in Eq.(15), we find

$$
0=a^{2}\left(\frac{1}{2} \dddot{k}_{2} z+\ddot{k}_{5}\right) \partial_{\mu} z+a^{-1}\left(\frac{d a}{d r}\right) \dot{k}_{1} \partial_{\mu} r
$$

from which it follows that $\dot{k}_{1}$ and $\ddot{k}_{5}$ and $\dddot{k}_{2}$ all vanish. Thus we have $k_{1}=c_{1}$ and $k_{5}=c_{5} t+c_{8}$ and $k_{2}=c_{2} t^{2}+c_{9} t+c_{10}$ for constants $c_{1}, c_{2}, c_{5}, c_{8}, c_{9}$, and $c_{10}$. 
To summarize, we have found that the general solution for $C_{\mu \nu}$ and $B_{\mu}$ takes the form

$$
\begin{aligned}
C_{\mu \nu} & =c_{1} h_{\mu \nu}+\left(c_{2} t^{2}+c_{9} t+c_{10}\right) \lambda_{\mu} \lambda_{\nu}+c_{3} \eta_{\mu} \eta_{\nu}+\left(c_{4} t+c_{7}\right) 2 \lambda_{(\mu} \eta_{\nu)} \\
B_{\mu} & =\left[\left(c_{2} t+\frac{1}{2} c_{9}\right) z+\left(c_{5} t+c_{8}\right)\right] \lambda_{\mu}+\left(c_{4} z+c_{6}\right) \eta_{\mu}
\end{aligned}
$$

It remains to find $A$. Since $B^{r}=0$, it follows from Eq.(16) that $\dot{A}=0$. Using the expressions of equations (64) and (65) in Eq.(17) for grad A, we find

$$
\partial_{\mu} A=2\left(c_{2} z+c_{5}\right) \partial_{\mu} z-c_{1} \partial_{\mu}\left(a^{-2}\right) .
$$

The general solution of Eq.(66) is

$$
A=c_{2} z^{2}+2 c_{5} z-c_{1} a^{-2}+c_{11}
$$

Finally, using the expressions of equations (64-67) in Eq.(4) we find that the most general Killing tensor of Melvin's magnetic universe takes the 4-dimensional form

$$
\begin{aligned}
X_{a b} & =c_{1} g_{a b}+c_{2}\left[z^{2} \tau_{a} \tau_{b}+z t \lambda_{(a} \tau_{b)}+t^{2} \lambda_{a} \lambda_{b}\right]+c_{3} \eta_{a} \eta_{b} \\
& +c_{4}\left[2 z \eta_{(a} \tau_{b)}+2 t \eta_{(a} \lambda_{b)}\right]+c_{5}\left[2 z \tau_{a} \tau_{b}+2 t \lambda_{(a} \tau_{b)}\right]+c_{6} 2 \eta_{(a} \tau_{b)} \\
& +c_{7} 2 \lambda_{(a} \eta_{b)}+c_{8} 2 \lambda_{(a} \tau_{b)}+c_{9}\left[z \lambda_{(a} \tau_{b)}+t \lambda_{a} \lambda_{b}\right]+c_{10} \lambda_{a} \lambda_{b}+c_{11} \tau_{a} \tau_{b}
\end{aligned}
$$

This expression can be simplified by noting that the Melvin boost Killing vector is given by $\psi^{a}:=t \lambda^{a}+z \tau^{a}$. The Killing tensor is then

$$
\begin{aligned}
X_{a b} & =c_{1} g_{a b}+c_{2} \psi_{a} \psi_{b}+c_{3} \eta_{a} \eta_{b}+2 c_{4} \eta_{(a} \psi_{b)}+2 c_{5} \psi_{(a} \tau_{b)}+2 c_{6} \eta_{(a} \tau_{b)} \\
& +2 c_{7} \lambda_{(a} \eta_{b)}+2 c_{8} \lambda_{(a} \tau_{b)}+c_{9} \psi_{(a} \lambda_{b)}+c_{10} \lambda_{a} \lambda_{b}+c_{11} \tau_{a} \tau_{b}
\end{aligned}
$$

Now each term in the sum is either the metric or the symmetrized product of two Killing vectors. Thus all the Killing tensors of Melvin's magnetic universe are trivial.

\section{KILLING TENSORS OF THE SCHWARZSCHILD METRIC}

The Schwarzschild vacuum solution is given by

$$
d s_{\text {Sch }}^{2}=-F d t^{2}+F^{-1} d r^{2}+r^{2}\left(d \vartheta^{2}+\sin ^{2} \vartheta d \varphi^{2}\right) .
$$


where the function $F$ is $F=1-(2 m / r)$. The metric admits four Killing vectors $\left(\tau^{a}, \alpha^{a}, \beta^{a}, \gamma^{a}\right)$; a timelike Killing vector $\tau^{a} \partial_{a}=\partial_{t}$, and three spacelike vectors which comprise the $\mathrm{SO}_{3}$ rotations

$$
\begin{aligned}
\alpha^{a} \partial_{a} & =\sin \varphi \partial_{\vartheta}+\cot \vartheta \cos \varphi \partial_{\varphi} \\
\beta^{a} \partial_{a} & =-\cos \varphi \partial_{\vartheta}+\cot \vartheta \sin \varphi \partial_{\varphi} \\
\gamma^{a} \partial_{a} & =\partial_{\varphi} \\
2 r^{2} & =\alpha^{a} \alpha_{a}+\beta^{a} \beta_{a}+\gamma^{a} \gamma_{a} .
\end{aligned}
$$

Each of the four Killing vectors is hypersurface orthogonal.

First we will find the Killing tensors of the 2-dimensional $r \vartheta$ surface. As before, we will use $c_{1}, c_{2}$ etc. to denote constants, and $k_{1}, k_{2}$ etc. to denote quantities that depend only on the coordinate associated with the Killing vector.

\section{A. An $r \vartheta$ surface}

The $r \vartheta$ 2-surface has metric

$$
g_{a b} d x^{a} d x^{b}=F^{-1} d r^{2}+r^{2} d \vartheta^{2}
$$

We have the $\vartheta$ coordinate Killing vector $\partial_{\vartheta}=\vartheta^{a} \partial_{a}$ for which $\epsilon=1$ and $V=r$ (note that $\vartheta^{a}$ is a symmetry of the 2-surface but not of the spacetime). The metric on the space orthogonal to the Killing vector is $h_{\mu \nu}=F^{-1} r_{, \mu} r,{ }_{\nu}$. Since the r-direction is a 1-dimensional line, it follows as before that the Killing tensor on that 1-dimensional space takes the form

$$
C_{\mu \nu}=k_{1} h_{\mu \nu}=k_{1} F^{-1} r_{, \mu} r_{\nu}
$$

for some $k_{1}(\vartheta)$. Equation (13) then becomes, with $B^{\mu} \rightarrow B^{r}$ only

$$
\dot{C}_{r r}=\dot{k}_{1} F^{-1}=-r^{2}\left[-\left(\frac{2 m}{r^{2}}\right) F^{-2} B^{r}+2 F^{-1} \frac{d B^{r}}{d r}\right]
$$

or

$$
\dot{k}_{1}=2 m F^{-1} B^{r}-2 r^{2} \frac{d B^{r}}{d r}
$$

The general solution for $B^{r}$ is

$$
B^{r}=-\left(\frac{\dot{k}_{1}}{2 m}\right) F+k_{2} F^{1 / 2}
$$


Equation (14) is automatically satisfied, while Eq.(15) becomes

$$
\left(\ddot{k}_{2}+\frac{1}{2} k_{2}\right) F^{-1 / 2}+\left(\frac{\dot{k}_{1}}{m}-\frac{\dddot{k_{1}}}{2 m}\right)+\frac{1}{2} k_{2} F^{1 / 2}-\frac{3}{2 m} \dot{k}_{1} F
$$

Here we have grouped terms so that each term is a coefficient independent of $r$ multiplied by a function of $r$, and the functions of $r$ are linearly independent. Thus each coefficient must vanish, which yields $k_{2}=0$ and $\dot{k}_{1}=0$, from which it follows that $k_{1}=c_{1}$ and $B^{r}=0$. It then follows from Eq.(7) that $A$ is independent of $\vartheta$. From Eq.(17) integration provides

$$
A(r)=\frac{c_{1}}{r^{2}}+c_{2}
$$

Using Eq.(4) we find that the general Killing tensor of the $r \vartheta$ surface is

$$
X_{a b}=c_{1} g_{a b}+c_{2} \vartheta_{a} \vartheta_{b}
$$

\section{B. A $\operatorname{tr} \vartheta$ surface}

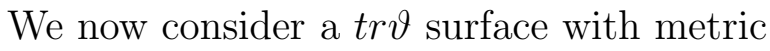

$$
g_{a b} d x^{a} d x^{b}=-F d t^{2}+F^{-1} d r^{2}+r^{2} d \vartheta^{2}
$$

The Killing field is $\tau^{a} \partial_{a}=\partial_{t}$, with $\epsilon=-1$ and $V=F^{1 / 2}$. Here $h_{\mu \nu}$ is the $g_{a b}$ of the previous subsection, while $C_{\mu \nu}$ is the $X_{a b}$ of the previous subsection. We have

$$
\begin{aligned}
h_{\mu \nu} & =F^{-1} r_{, \mu} r_{, \nu}+r^{2} \vartheta_{, \mu} \vartheta_{{ }_{\nu}} \\
C_{\mu \nu} & =k_{1} h_{\mu \nu}+k_{2} \vartheta_{\mu} \vartheta_{\nu} .
\end{aligned}
$$

With $B^{\mu} \rightarrow\left(B^{r}, B^{\vartheta}\right)$, the $r r, \vartheta \vartheta$, and $r \vartheta$ components of Eq.(13) are then, respectively

$$
\begin{aligned}
\dot{k}_{1} F^{-1} & =2 \partial_{r} B^{r}-\frac{2 m}{r^{2}} F^{-1} B^{r} \\
\dot{k}_{1} r^{2}+\dot{k}_{2} r^{4} & =2 r^{2} F \partial_{\vartheta} B^{\vartheta}+2 r F B^{r} \\
0 & =r^{2} \partial_{r} B^{\vartheta}+F^{-1} \partial_{\vartheta} B^{r}
\end{aligned}
$$

From Eq.(82) we find that $B^{r}$ must take the form

$$
B^{r}=F^{1 / 2} H(t, \vartheta)+\frac{1}{2} \dot{k}_{1}\left\{[r-6 m]+3 m F^{1 / 2} \ln \left[\frac{r}{m}\left(1+F^{1 / 2}\right)-1\right]\right\}
$$


for some function $H(t, \vartheta)$. Using the expression of Eq. (85) in Eq. (84) we find

$$
\partial_{r} B^{\vartheta}=-r^{-2} F^{-1 / 2} \partial_{\vartheta} H
$$

Upon integration, it follows that $B^{\vartheta}$ is

$$
B^{\vartheta}=Q(t, \vartheta)-\frac{1}{m} F^{1 / 2} \partial_{\vartheta} H
$$

for integration function $Q(t, \vartheta)$. Substituting the expressions for $B^{r}$ and $B^{\vartheta}$ from equations (85) and (87) into Eq. (83) provides

$$
\begin{aligned}
\dot{k}_{1}+\dot{k}_{2} r^{2} & =\frac{2 F}{r}\left[F^{1 / 2} H(t, \vartheta)+\frac{1}{2} \dot{k}_{1}\left\{[r-6 m]+3 m F^{1 / 2} \ln \left[\frac{r}{m}\left(1+F^{1 / 2}\right)-1\right]\right\}\right] \\
& +2 F\left[\partial_{\vartheta} Q-\frac{1}{m} F^{1 / 2} \frac{\partial^{2} H}{\partial \vartheta^{2}}\right] .
\end{aligned}
$$

Note that each term in Eq. (88) is a function of $r$ multiplied by a coefficient that is independent of $r$. Since the function of $r$ that has the logarithmic term in Eq.(88) is linearly independent of the other functions of $r$, its coefficient must vanish. This implies that $\dot{k}_{1}=0$ and that $k_{1}=c_{1}$ for some constant $c_{1}$. Equation (88) then simplifies to

$$
0=-\frac{1}{2} \dot{k}_{2}\left(\frac{r^{2}}{F}\right)+H\left(\frac{F^{1 / 2}}{r}\right)+\partial_{\vartheta} Q-\frac{1}{m} \frac{\partial^{2} H}{\partial \vartheta^{2}}\left(F^{1 / 2}\right) .
$$

Here terms are grouped so that each term is a coefficient independent of $r$ multiplied by a function of $r$, and so that the functions of $r$ are linearly independent. It therefore follows that each of the coefficients vanishes. We have $\dot{k}_{2}=0, H=0$, and $\partial_{\vartheta} Q=0$. Therefore $k_{2}=c_{2}$ for some constant $c_{2}$, and the components of vector field $B^{\mu}$ are

$$
B^{r}=0, \quad B^{\vartheta}=k_{3}(t)
$$

for some function $k_{3}(t)$. Equivalently

$$
B_{\mu}=k_{3} r^{2} \partial_{\mu} \vartheta
$$

Since $k_{1}=c_{1}$ and $k_{2}=c_{2}$, it follows from Eq.(81) that tensor $C_{\mu \nu}$ takes the form

$$
C_{\mu \nu}=c_{1} h_{\mu \nu}+c_{2} r^{4} \vartheta_{, \mu} \vartheta_{, \nu}
$$

Upon using equations (91) and (92) in Eq.(14) we find that

$$
\partial_{[\mu}\left(F^{-1} r^{2} \dot{k}_{3} \partial_{\nu]} \vartheta\right)=0
$$


from which it follows that $\dot{k}_{3}=0$ and therefore that $k_{3}=c_{3}$ for some constant $c_{3}$. Thus, from Eq.(91) we have

$$
B_{\mu}=c_{3} r^{2} \partial_{\mu} \vartheta
$$

Equation (15) is identically satisfied by equations (92) and (94).

Since $B^{r}=0$ it follows from Eq.(16) that $\dot{A}=0$. Using equations (92) and (94) in Eq.(17) we obtain

$$
\partial_{\mu} A=-c_{1} \partial_{\mu}\left(F^{-1}\right)
$$

It then follows that

$$
A=c_{4}-c_{1} F^{-1}
$$

for some constant $c_{4}$. Finally, using the results of equations (92), (94) and (96) in Eq.(41), we find that the general Killing tensor of a $\operatorname{tr} \theta$ surface is

$$
X_{a b}=c_{1} g_{a b}+c_{2} \vartheta_{a} \vartheta_{b}+2 c_{3} \vartheta_{(a} \tau_{b)}+c_{4} \tau_{a} \tau_{b}
$$

\section{The Schwarzschild metric}

We are now ready to treat the full Schwarzschild metric by adding the axial $\gamma^{a}$ Killing vector to the metric of the previous subsection. We have

$$
\begin{aligned}
& h_{\mu \nu}=-F t_{, \mu} t_{, \nu}+F^{-1} r_{, \mu} r_{, \nu}+r^{2} \vartheta_{, \mu} \vartheta_{, \nu} \\
& C_{\mu \nu}=k_{1} h_{\mu \nu}+k_{2} \vartheta_{\mu} \vartheta_{\nu}+2 k_{3} \vartheta_{(\mu} \tau_{\nu)}+k_{4} \tau_{\mu} \tau_{\nu}
\end{aligned}
$$

The $\gamma^{a}$ Killing vector has $\epsilon=1$ and $V=r \sin \vartheta$. Equation (13) for $\dot{C}_{\mu \nu}$ then becomes

$$
\begin{aligned}
-\dot{k}_{1} F+\dot{k}_{4} F^{2} & =r^{2} \sin ^{2} \vartheta\left(B^{r} \partial_{r} F+2 F \partial_{t} B^{t}\right) \\
\dot{k}_{1} F^{-1} & =r^{2} \sin ^{2} \vartheta\left(F^{-2} B^{r} \partial_{r} F-2 F^{-1} \partial_{r} B^{r}\right) \\
\dot{k}_{1} r^{2}+\dot{k}_{2} r^{4} & =-2 r^{3} \sin ^{2} \vartheta\left(B^{r}+r \partial_{\vartheta} B^{\vartheta}\right) \\
0 & =F^{-1} \partial_{t} B^{r}-F \partial_{r} B^{t} \\
-\dot{k}_{3} r^{2} F & =r^{2} \sin ^{2} \vartheta\left(F \partial_{\vartheta} B^{t}-r^{2} \partial_{t} B^{\vartheta}\right) \\
0 & =r^{2} \partial_{r} B^{\vartheta}+F^{-1} \partial_{\vartheta} B^{r}
\end{aligned}
$$

Equation (101) can be rewritten as

$$
\partial_{r}\left(F^{-1 / 2} B^{r}\right)=-\frac{\dot{k}_{1}}{2 F^{1 / 2} r^{2} \sin ^{2} \vartheta}
$$


with integral

$$
B^{r}=G(t, \vartheta, \varphi) F^{1 / 2}-\frac{\dot{k}_{1}}{2 m \sin ^{2} \vartheta} F
$$

for integration function $G(t, \vartheta, \varphi)$. Using the result of Eq.(107) in Eq.(105), we find

$$
\partial_{r} B^{\vartheta}=-\frac{\partial_{\vartheta} G}{r^{2} F^{1 / 2}}-\frac{\dot{k}_{1} \cos \vartheta}{m r^{2} \sin ^{3} \vartheta} .
$$

Integration provides

$$
B^{\vartheta}=H(t, \vartheta, \varphi)-\left(\partial_{\vartheta} G\right) \frac{1}{m} F^{1 / 2}+\frac{\dot{k}_{1} \cos \vartheta}{m r \sin ^{3} \vartheta}
$$

with integration function $H(t, \vartheta, \varphi)$. Now using equations (107) and (109) in Eq.(102) we obtain

$0=-3 \dot{k}_{1}-\left(\dot{k}_{2}+\partial_{\vartheta} H 2 \sin ^{2} \vartheta\right) r^{2}+\frac{\dot{k}_{1}}{m}\left(\frac{6}{\sin ^{2} \vartheta}-3\right) r-2 \sin ^{2} \vartheta G\left(r F^{1 / 2}\right)+\frac{2}{m} \sin ^{2} \vartheta\left(\frac{\partial^{2} G}{\partial \vartheta^{2}}\right) r^{2} F^{1 / 2}$

Here we have grouped terms so that each term is a coefficient independent of $r$ multiplied by a function of $r$, and so that the functions of $r$ are linearly independent. It therefore follows that each coefficient vanishes. Thus $G=\dot{k}_{1}=0$ and

$$
\partial_{\vartheta} H=-\frac{\dot{k}_{2}}{2 \sin ^{2} \vartheta} \text {. }
$$

From the vanishing of $G$ and $\dot{k}_{1}$ it follows that $B^{r}$ vanishes, and that $k_{1}=c_{1}$ for some constant $c_{1}$ and that $B^{\vartheta}=H$. From Eq.(111) it follows that $B^{\vartheta}$ takes the form

$$
B^{\vartheta}=I(t, \varphi)+\frac{\dot{k}_{2}}{2} \cot \vartheta
$$

for some function $I(t, \varphi)$. Using Eq.(112), along with $B^{r}=0$ and $\dot{k}_{1}=0$, reduces equations (100, 105) to the following:

$$
\begin{aligned}
\partial_{t} B^{t} & =\frac{\dot{k}_{4} F}{2 r^{2} \sin ^{2} \vartheta} \\
\partial_{r} B^{t} & =0 \\
\partial_{\vartheta} B^{t} & =r^{2} F^{-1} \partial_{t} I-\frac{\dot{k}_{3}}{\sin ^{2} \vartheta}
\end{aligned}
$$

Applying $\partial_{r}$ to Eq.(113) and using Eq.(114) yields $\dot{k}_{4}=0$. Therefore $B^{t}$ is independent of $t$ and $k_{4}=c_{2}$ for some constant $c_{2}$. Now applying $\partial_{r}$ to Eq.(115) and using Eq.(114) it follows that $\partial_{t} I=0$ and $I=k_{5}$ for some function $k_{5}(\varphi)$. Thus $B^{\vartheta}$ becomes

$$
B^{\vartheta}=k_{5}+\frac{\dot{k}_{2}}{2} \cot \vartheta
$$


Integrating Eq.(115) yields

$$
B^{t}=k_{6}+\dot{k}_{3} \cot \vartheta
$$

for some function $k_{6}(\varphi)$. In summary, we have found that $B_{\mu}$ and $C_{\mu \nu}$ take the form

$$
\begin{aligned}
B_{\mu} & =\left(k_{6}+\dot{k}_{3} \cot \vartheta\right) \tau_{\mu}+\left(k_{5}+\frac{\dot{k}_{2}}{2} \cot \vartheta\right) \vartheta_{\mu} \\
C_{\mu \nu} & =c_{1} h_{\mu \nu}+k_{2} \vartheta_{\mu} \vartheta_{\nu}+2 k_{3} \vartheta_{(\mu} \tau_{\nu)}+c_{2} \tau_{\mu} \tau_{\nu}
\end{aligned}
$$

We now impose the integrability conditions of equations (14) and (15) on the expressions above for $B_{\mu}$ and $C_{\mu \nu}$. From equations (118) and (119), with $\epsilon=1$ and $V=r \sin \vartheta$, we find

$$
\begin{aligned}
V^{-2} \dot{B}_{\mu}+V^{-3} C_{\mu \nu} \partial^{\nu} V & =c_{1} V^{-3} \partial_{\mu} V-\frac{F}{r^{2} \sin ^{2} \vartheta}\left[\dot{k}_{6}+\left(\ddot{k}_{3}+k_{3}\right) \cot \vartheta\right] \partial_{\mu} t \\
& +\frac{1}{\sin ^{2} \vartheta}\left[\dot{k}_{5}+\left(\frac{\ddot{k}_{2}}{2}+k_{2}\right) \cot \vartheta\right] \partial_{\mu} \vartheta
\end{aligned}
$$

The integrability condition given in Eq.(14) is the statement that the right hand side of Eq.(120) is curl-free. From this it follows that, for the term in Eq.(120) multiplying $\partial_{\mu} t$, the quantity in square brackets vanishes. That is, we have $\dot{k}_{6}=0$ and $\ddot{k}_{3}+k_{3}=0$. Thus

$$
\begin{aligned}
& k_{6}=c_{3} \\
& k_{3}=c_{4} \cos \varphi+c_{5} \sin \varphi
\end{aligned}
$$

for constants $c_{3}, c_{4}$, and $c_{5}$.

From equations (118), (120), (121), and (122) it follows that

$$
\ddot{B}_{\mu}+V^{-1} \dot{C}_{\mu \nu} \partial^{\nu} V-\epsilon V^{2} \partial_{\mu}\left(V^{-1} B^{\nu} \partial_{\nu} V\right)=\left[\ddot{k}_{5}+k_{5}+\left(\frac{\dddot{k}_{2}}{2}+2 \dot{k}_{2}\right) \cot \vartheta\right] r^{2} \partial_{\mu} \vartheta
$$

The integrability condition of Eq.(15) states that the right hand side of Eq.(123) vanishes. Therefore $\ddot{k}_{5}+k_{5}=0$ and $\dddot{k}_{2}+4 \dot{k}_{2}=0$, and thus

$$
\begin{aligned}
& k_{5}=c_{6} \cos \varphi+c_{7} \sin \varphi \\
& k_{2}=c_{8} \cos 2 \varphi+c_{9} \sin 2 \varphi+c_{10}
\end{aligned}
$$

for constants $c_{6}, c_{7}, c_{8}, c_{9}$, and $c_{10}$. We find that the general solution for $B_{\mu}$ and $C_{\mu \nu}$ is

$$
\begin{aligned}
B_{\mu} & =\left[c_{3}+\left(-c_{4} \sin \varphi+c_{5} \cos \varphi\right) \cot \vartheta\right] \tau_{\mu} \\
& +\left[c_{6} \cos \varphi+c_{7} \sin \varphi+\left(-c_{8} \sin 2 \varphi+c_{9} \cos 2 \varphi\right) \cot \vartheta\right] \vartheta_{\mu} \\
C_{\mu \nu} & =c_{1} h_{\mu \nu}+\left(c_{8} \cos 2 \varphi+c_{9} \sin 2 \varphi+c_{10}\right) \vartheta_{\mu} \vartheta_{\nu} \\
& +2\left(c_{4} \cos \varphi+c_{5} \sin \varphi\right) \vartheta_{(\mu} \tau_{\nu)}+c_{2} \tau_{\mu} \tau_{\nu}
\end{aligned}
$$


It remains to find $A$. Using equations (126) and (127) in Eq.(17) we obtain

$$
\begin{aligned}
\partial_{\mu} A & =-2 c_{1} V^{-3} \partial_{\mu} V \\
& -\frac{2}{\sin ^{2} \vartheta}\left[-c_{6} \sin \varphi+c_{7} \cos \varphi+\left(-c_{8} \cos 2 \varphi-c_{9} \sin 2 \varphi+c_{10}\right) \cot \vartheta\right] \partial_{\mu} \vartheta
\end{aligned}
$$

with general solution

$$
A=c_{1} V^{-2}+2\left(-c_{6} \sin \varphi+c_{7} \cos \varphi\right) \cot \vartheta+\left(-c_{8} \cos 2 \varphi-c_{9} \sin 2 \varphi+c_{10}\right) \cot ^{2} \vartheta+k_{7}
$$

for some function $k_{7}(\varphi)$. Imposing Eq.(16) on Eq.(129) we find that $\dot{k}_{7}=0$ and therefore that $k_{7}=c_{11}$ for some constant $c_{11}$. The solution for $A$ becomes

$$
A=c_{1} V^{-2}+2\left(-c_{6} \sin \varphi+c_{7} \cos \varphi\right) \cot \vartheta+\left(-c_{8} \cos 2 \varphi-c_{9} \sin 2 \varphi+c_{10}\right) \cot ^{2} \vartheta+c_{11}
$$

Using equations (126), (127), and (130) in Eq.(44), and grouping terms according to their constant coefficient, we find that the general Schwarzschild Killing tensor is

$$
\begin{aligned}
X_{a b} & =c_{1}\left(h_{a b}+V^{-2} \gamma_{a} \gamma_{b}\right)+c_{2} \tau_{a} \tau_{b}+c_{3} 2 \tau_{(a} \gamma_{b)} \\
& +c_{4}\left[2 \cos \varphi \vartheta_{(a} \tau_{b)}-2 \sin \varphi \cot \vartheta \gamma_{(a} \tau_{b}\right] \\
& +c_{5}\left[2 \sin \varphi \vartheta_{(a} \tau_{b)}+2 \cos \varphi \cot \vartheta \gamma_{(a} \tau_{b)}\right]+c_{6}\left[2 \cos \varphi \vartheta_{(a} \gamma_{b)}-2 \sin \varphi \cot \vartheta \gamma_{a} \gamma_{b}\right] \\
& +c_{7}\left[2 \sin \varphi \vartheta_{(a} \gamma_{b)}+2 \cos \varphi \cot \vartheta \gamma_{a} \gamma_{b}\right] \\
& +c_{8}\left[\cos 2 \varphi \vartheta_{a} \vartheta_{b}-2 \sin 2 \varphi \cot \vartheta \vartheta_{(a} \gamma_{b)}-\cos 2 \varphi \cot ^{2} \vartheta \gamma_{a} \gamma_{b}\right] \\
& +c_{9}\left[\sin 2 \varphi \vartheta_{a} \vartheta_{b}+2 \cos 2 \varphi \cot \vartheta \vartheta_{(a} \gamma_{b)}-\sin 2 \varphi \cot ^{2} \vartheta \gamma_{a} \gamma_{b}\right] \\
& +c_{10}\left[\vartheta_{a} \vartheta_{b}+\cot ^{2} \vartheta \gamma_{a} \gamma_{b}\right]+c_{11} \gamma_{a} \gamma_{b}
\end{aligned}
$$

We now rewrite this expression for the Killing tensor in terms of Killing vectors and the metric. We have

$$
\begin{aligned}
g_{a b} & =h_{a b}+V^{-2} \gamma_{a} \gamma_{b}, \\
\alpha_{a} & =\sin \varphi \vartheta_{a}+(\cot \vartheta \cos \varphi) \gamma_{a}, \\
\beta_{a} & =-\cos \varphi \vartheta_{a}+(\cot \vartheta \sin \varphi) \gamma_{a},
\end{aligned}
$$

which yields

$$
\begin{aligned}
\alpha_{a} \alpha_{b}+\beta_{a} \beta_{b} & =\vartheta_{a} \vartheta_{b}+\cot ^{2} \vartheta \gamma_{a} \gamma_{b} \\
\alpha_{a} \alpha_{b}-\beta_{a} \beta_{b} & =\cos 2 \varphi\left(-\vartheta_{a} \vartheta_{b}+\cot ^{2} \vartheta \gamma_{a} \gamma_{b}\right)+2 \cot \vartheta \sin 2 \varphi \vartheta_{(a} \gamma_{b)} \\
2 \alpha_{(a} \beta_{b)} & =\sin 2 \varphi\left(-\vartheta_{a} \vartheta_{b}+\cot ^{2} \vartheta \gamma_{a} \gamma_{b}\right)-2 \cot \vartheta \cos 2 \varphi \vartheta_{(a} \gamma_{b)}
\end{aligned}
$$


Using the three equations above, we can rewrite $X_{a b}$ as

$$
\begin{aligned}
X_{a b} & =c_{1} g_{a b}+c_{2} \tau_{a} \tau_{b}+c_{3} 2 \tau_{(a} \gamma_{b)}-2 c_{4} \beta_{(a} \tau_{b)}+2 c_{5} \alpha_{(a} \tau_{b)}-2 c_{6} \beta_{(a} \gamma_{b)}+2 c_{7} \alpha_{(a} \gamma_{b)} \\
& -c_{8}\left(\alpha_{a} \alpha_{b}-\beta_{a} \beta_{b}\right)-2 c_{9} \alpha_{(a} \beta_{b)}+c_{10}\left(\alpha_{a} \alpha_{b}+\beta_{a} \beta_{b}\right)+c_{11} \gamma_{a} \gamma_{b} .
\end{aligned}
$$

Finally, regrouping terms we have

$$
\begin{aligned}
X_{a b} & =c_{1} g_{a b}+c_{2} \tau_{a} \tau_{b}+c_{3} 2 \tau_{(a} \gamma_{b)}-2 c_{4} \beta_{(a} \tau_{b)}+2 c_{5} \alpha_{(a} \tau_{b)}-2 c_{6} \beta_{(a} \gamma_{b)}+2 c_{7} \alpha_{(a} \gamma_{b)} \\
& +\left(c_{10}-c_{8}\right) \alpha_{a} \alpha_{b}+\left(c_{10}+c_{8}\right) \beta_{a} \beta_{b}-2 c_{9} \alpha_{(a} \beta_{b)}+c_{11} \gamma_{a} \gamma_{b} .
\end{aligned}
$$

Thus, we have written the general Schwarzschild Killing tensor as a sum of terms where each term is either the metric or a product of Killing vectors. Therefore all Killing tensors of the Schwarzschild spacetime are trivial.

\section{CONCLUSIONS}

The method used above consists of applying equations (13, 17$)$ to find the Killing tensor in n-dimensions, using the equations in n-1 dimensions. For the Melvin metric and the Schwarzschild metric this is done three times, going from a 1-dimensional space to the 4dimensional spacetime.

The method developed here for finding Killing tensors could be used on a wide variety of spacetimes where there are symmetries. It requires less computation than an attack on the full Killing tensor equations, and it can be used even when the spacetime is not algebraically special. The method could also be generalized in various ways. The equations for a Killing-Yano tensor [6] could be treated in an analogous way and should result in a method simpler than a straightforward attempt to solve the Killing-Yano equations. Finally the method might have a useful generalization to the case where the Killing vector is not hypersurface orthogonal. In that case one would expect to get more complicated equations that involve not only the norm of the Killing field but also the twist. However, it is in just such spacetimes (i.e. Kerr) that known examples of nontrivial Killing tensors exist. So an investigation along those lines might be useful.

[1] B. Carter, Hamilton-Jacobi and Schrödinger separable solutions of Einstein's equations, Commun. Math. Phys. 10, 280 (1968). 
[2] P. Sommers, On Killing tensors and constants of motion, J. Math. Phys. 14, 787 (1973).

[3] Exact Solutions of Einstein's Field Equations, Eds. D. Kramer, H. Stephani, E. Herlt, M. MacCallum and E. Schmutzer, 2nd Ed. (Cambridge University Press, Cambridge, U.K. 2003).

[4] R. Geroch, J. Math. Phys. A Method for Generating New Solutions of Einstein's Equation. II, 13, 394 (1972).

[5] M.A. Melvin, Pure Magnetic and Electric Geons, Phys. Lett. 8, 65 (1964).

[6] C.D. Collinson, On the Relationship between Killing Tensors and Killing-Yano Tensors, Int. Jour. Theor. Phys. 15, 311 (1976). 


\title{
Killing Tensors and Symmetries
}

\author{
David Garfinkle \\ Physics Department, Oakland University, Rochester, MI \\ E.N. Glass \\ Physics Department, University of Michigan, Ann Arbor, MI
}

(Dated: March 11, 2010)

\begin{abstract}
A new method is presented for finding Killing tensors in spacetimes with symmetries. The method is used to find all the Killing tensors of Melvin's magnetic universe and the Schwarzschild vacuum. We show that they are all trivial. The method requires less computation than solving the full Killing tensor equations directly, and it can be used even when the spacetime is not algebraically special

PACS numbers: 04.20.Cv, 04.20.Jb
\end{abstract}

\section{INTRODUCTION}

Killing tensors are useful because, like Killing vectors, they provide conserved quantities for geodesic motion, most famously in the Kerr metric where the Killing tensor gives rise to the Carter constant [1]. However, it is much more difficult to find Killing tensors than Killing vectors. In 4 spacetime dimensions, the equation for a Killing tensor becomes 20 partial differential equations for 10 functions of 4 variables. Most of the known results on Killing tensors come from the fact that the Killing tensor equation simplifies in certain classes of algebraically special spacetimes [2, 3]. However, equations in general relativity also often simplify in the presence of symmetry [4]. We will show that the Killing tensor equation simplifies when a spacetime possesses a hypersurface orthogonal Killing vector, and that this simplification provides an effective method for finding Killing tensors. We will apply this method to Melvin's magnetic universe [5] and the Schwarzschild vacuum solution. It is shown that all of their Killing tensors are trivial in the sense that they are either the metric or the symmetrized product of Killing vectors. The method is presented in section 2. It is applied to Melvin's magnetic universe in section 3 and the Schwarzschild vacuum in section 4. Conclusions are given in section 5 . 
Notation: Lower case Latin indices, $B^{a}$, range over n-dimensions. Greek indices, $B^{\mu}$, range over n-1 dimensions. For Killing vector $\xi^{a}$ an overdot will denote a Lie derivative, $\dot{A}:=\mathcal{L}_{\xi} A$.

\section{THE KILLING TENSOR METHOD}

A Killing tensor (of order 2) is a symmetric tensor $X_{a b}$ that satisfies

$$
\nabla_{(a} X_{b c)}=0
$$

Now suppose that the spacetime has a hypersurface orthogonal Killing vector $\xi^{a}$. Define $V$ such that

$$
\xi^{a} \xi_{a}=\epsilon V^{2}
$$

where $\epsilon= \pm 1$. Then the metric in directions orthogonal to $\xi^{a}$ is given by

$$
h_{a b}=g_{a b}-\epsilon V^{-2} \xi_{a} \xi_{b}
$$

One can use $h_{b}^{a}$ as a projection operator to project any tensor in directions orthogonal to $\xi^{a}$. In particular, the Killing tensor can be decomposed as

$$
X_{a b}=A \xi_{a} \xi_{b}+2 B_{(a} \xi_{b)}+C_{a b}
$$

where $B_{a}$ and $C_{a b}$ are orthogonal to $\xi^{a}$. The Killing tensor equation for this decomposition is

$$
\nabla_{(a} A \xi_{b} \xi_{c)}+2 \nabla_{(a} B_{b} \xi_{c)}+\nabla_{(a} C_{b c)}=0 .
$$

Projecting the Killing tensor equation using all combinations of $h^{a}{ }_{b}$ and $\xi^{a}$ yields the following

$$
\begin{aligned}
D_{(a} C_{b c)} & =0, \\
\mathcal{L}_{\xi} A & =-2 V^{-1} B^{a} D_{a} V, \\
\mathcal{L}_{\xi} B_{a} & =-V^{-1} C_{a b} D^{b} V-\frac{1}{2} \epsilon V^{2} D_{a} A, \\
\mathcal{L}_{\xi} C_{a b} & =-2 \epsilon V^{2} D_{(a} B_{b)} .
\end{aligned}
$$

Here $D_{a}$ is the derivative operator associated with the metric $h_{a b}$ and $\mathcal{L}_{\xi}$ denotes the Lie derivative with respect to Killing vector $\xi^{a}$. Solving Eq.(8) for $D_{a} A$ yields

$$
D_{a} A=-(2 / \epsilon)\left[V^{-2} \mathcal{L}_{\xi} B_{a}+V^{-3} C_{a b} D^{b} V\right]
$$


The left hand side of Eq.(10) is curl-free and thus the curl of the right hand side must vanish. Hence

$$
D_{[a}\left(V^{-2} \mathcal{L}_{\xi} B_{b]}\right)+D_{[a}\left(V^{-3} C_{b] c} D^{c} V\right)=0
$$

Furthermore, since $\mathcal{L}_{\xi}$ commutes with $D_{a}$ it follows that $\mathcal{L}_{\xi}$ of the right hand side of Eq.(10) equals $D_{a}$ of the right hand side of Eq.(7), and therefore

$$
\mathcal{L}_{\xi} \mathcal{L}_{\xi} B_{a}+V^{-1}\left(D^{b} V\right) \mathcal{L}_{\xi} C_{a b}-\epsilon V^{2} D_{a}\left(V^{-1} B^{b} D_{b} V\right)=0 .
$$

Equations (11) and (12) provide the integrability conditions for equations (71) and (10).

These equations are most easily implemented in a coordinate system adapted to the Killing vector. Choose a coordinate system $\left(y, x^{\mu}\right)$ such that $x^{\mu}$ are coordinates on the surface orthogonal to the Killing vector and $\mathcal{L}_{\xi}$ is simply a partial derivative with respect to $y$. Use $\partial_{\mu}$ or a comma to denote a derivative with respect to the $x^{\mu}$ coordinates. The Latin indices in this section are n-dimensional, and the method below projects objects and equations down to n-1 dimensions with Greek indices.

Equation (91) becomes

$$
\dot{C}_{\mu \nu}=-\epsilon V^{2}\left(B^{\alpha} \partial_{\alpha} h_{\mu \nu}+h_{\alpha \nu} \partial_{\mu} B^{\alpha}+h_{\mu \alpha} \partial_{\nu} B^{\alpha}\right)
$$

while integrability conditions (11) and (12) become

$$
\begin{aligned}
\partial_{[\mu}\left(V^{-2} \dot{B}_{\nu]}\right)+\partial_{[\mu}\left(V^{-3} C_{\nu] \alpha} \partial^{\alpha} V\right) & =0 \\
\ddot{B}_{\mu}+V^{-1} \dot{C}_{\mu \nu} \partial^{\nu} V-\epsilon V^{2} \partial_{\mu}\left(V^{-1} B^{\nu} \partial_{\nu} V\right) & =0 .
\end{aligned}
$$

Equations (7) and (10) for $A$ are written as

$$
\begin{aligned}
\dot{A} & =-2 V^{-1} B^{\mu} \partial_{\mu} V \\
\partial_{\mu} A & =-(2 / \epsilon)\left(V^{-2} \dot{B}_{\mu}+V^{-3} C_{\mu \nu} \partial^{\nu} V\right)
\end{aligned}
$$

Note that equations (13-17) can be evaluated without ever having to calculate a Christoffel symbol.

The complete set of Killing tensors can be found as follows: first solve Eq.(66) for the most general $C_{\mu \nu}$. Note that this general solution will contain arbitrary "constants" that are really functions of the Killing coordinate. Now using the general $C_{\mu \nu}$, find the most general $B_{\mu}$ satisfying Eq.(13). Then restrict this general solution by demanding that equations (14) and (15) also be satisfied. Finally, solve equations (16) and (17) for $A$. 


\section{KILLING TENSORS OF THE MELVIN METRIC}

Melvin's magnetic universe is a static, cylindrically symmetric, Petrov type D solution of the Einstein-Maxwell equations. Its metric is usually written as

$$
d \tilde{s}_{\mathrm{Mel}}^{2}=a^{2}\left(-d \tilde{t}^{2}+d \rho^{2}+d \tilde{z}^{2}\right)+\left(\rho^{2} / a^{2}\right) d \tilde{\phi}^{2}
$$

where the function $a$ is

$$
a=1+\frac{1}{4} B_{0}^{2} \rho^{2}
$$

The constant $B_{0}$ is the value of the magnetic field on the $\rho=0$ axis. Define coordinates $t=\left(B_{0} / 2\right) \tilde{t}, r=\left(B_{0} / 2\right) \rho, z=\left(B_{0} / 2\right) \tilde{z}, \phi=\tilde{\phi}$. In terms of these coordinates we have

$$
a=1+r^{2}
$$

while the metric becomes $d \tilde{s}_{\text {Mel }}^{2}=\left(4 / B_{0}^{2}\right) d s_{\text {Mel }}^{2}$ with

$$
d s_{\text {Mel }}^{2}=a^{2}\left(-d t^{2}+d r^{2}+d z^{2}\right)+\left(r^{2} / a^{2}\right) d \phi^{2} .
$$

Since $d \tilde{s}_{\text {Mel }}^{2}$ and $d s_{\text {Mel }}^{2}$ differ only by an overall constant scale, they have the same Killing

vectors and Killing tensors. For simplicity, we will work with metric $d s_{\mathrm{Mel}}^{2}$. Since the metric components are independent of $t, \phi$, and $z$, it follows that there are Killing vectors for each of these coordinate directions. They are denoted by $(t, \phi, z) \rightarrow\left(\tau^{\mu}, \eta^{\mu}, \lambda^{\mu}\right)$ respectively. Each of these Killing vectors is hypersurface orthogonal. In addition, the metric has boost symmetry in the $t z$ plane, with corresponding Killing field $t \lambda^{a}+z \tau^{a}$.

We will use the method of the previous section to work out the Killing tensors of Melvin's magnetic universe. First we will find the Killing tensors of the 2-dimensional $r z$ surface, then use these to find the Killing tensors of the 3-dimensional $r z \phi$ surface, and finally find the Killing tensors of the 4-dimensional Melvin metric.

We will use $c_{1}, c_{2}$ etc. to denote constants, and $k_{1}, k_{2}$ etc. to denote quantities that depend only on the coordinate associated with the Killing vector.

\section{A. An $r z$ surface}

The $r z 2$-surface has a metric of form

$$
g_{a b} d x^{a} d x^{b}=a^{2}\left(d r^{2}+d z^{2}\right)
$$


We have a $z$ coordinate Killing vector $\lambda^{\mu}$ for which $V=a$ and $\epsilon=1$, and a 1-dimensional metric $h_{\mu \nu}=a^{2} r_{, \mu} r_{, \nu}$ orthogonal to the Killing vector. Since the r-direction is a 1dimensional line, it follows that the Killing tensor must take the form $C_{\mu \nu}=F h_{\mu \nu}$ for some scalar $F$. It then follows from Eq.(6) that $F$ is independent of $r$. We therefore have

$$
C_{\mu \nu}=k_{1} h_{\mu \nu}=k_{1} a^{2} r_{, \mu} r_{, \nu}
$$

for some $k_{1}(z)$. Equation (13) then becomes, with $B^{\mu} \rightarrow B^{r}$ only

$$
\dot{C}_{r r}=\dot{k}_{1} a^{2}=-a^{2}\left(\frac{d a^{2}}{d r} B^{r}+2 a^{2} \partial_{r} B^{r}\right)
$$

or

$$
\dot{k}_{1}=-2 a^{2}\left(\frac{d B^{r}}{d r}+\frac{1}{a} \frac{d a}{d r} B^{r}\right)
$$

The general solution for $B^{r}$ is

$$
B^{r}=a^{-1}\left(k_{2}-\frac{\dot{k}_{1}}{2} \arctan r\right)
$$

Equation (14) is automatically satisfied, while Eq.(15) becomes

$$
0=\left(-\frac{1}{2} \dddot{k}_{1}-2 \dot{k}_{1}\right) a^{2} \arctan r+3 \dot{k}_{1} a \arctan r+\left(\ddot{k}_{2}+4 k_{2}\right) a^{2}+3 \dot{k}_{1} r-6 k_{2} a
$$

Here we have grouped terms so that each term is a coefficient independent of $r$ multiplied by a function of $r$ and the functions of $r$ are linearly independent. Thus each coefficient must vanish, which yields $k_{2}=0$ and $\dot{k}_{1}=0$, from which it follows that $k_{1}=c_{1}$ and $B^{r}=0$. It then follows from Eq.(77) that $A$ is independent of $z$. From Eq.(17) it follows that

$$
A(r)=c_{2}+c_{1} a^{-2}
$$

Using Eq.(4) we find that the general Killing tensor of the $r z$ surface is

$$
X_{a b}=c_{1} g_{a b}+c_{2} \lambda_{a} \lambda_{b}
$$

\section{B. An $r z \phi$ surface}

We now consider an $r z \phi$ surface with metric

$$
g_{a b} d x^{a} d x^{b}=a^{2}\left(d r^{2}+d z^{2}\right)+\left(r^{2} / a^{2}\right) d \phi^{2} .
$$


The Killing field is $\eta^{a}$, with $\epsilon=1$ and $V=r / a$. Here $h_{\mu \nu}$ is the $g_{a b}$ of the previous subsection, while $C_{\mu \nu}$ is the $X_{a b}$ of the previous subsection. We have

$$
\begin{aligned}
& h_{\mu \nu}=a^{2}\left(r,_{\mu} r,_{\nu}+z,,_{\mu} z,_{\nu}\right) \\
& C_{\mu \nu}=k_{1} h_{\mu \nu}+k_{2} \lambda_{\mu} \lambda_{\nu} .
\end{aligned}
$$

With $B^{\mu} \rightarrow\left(B^{r}, B^{z}\right)$, the $r r, z z$, and $r z$ components of Eq.(13) are then, respectively

$$
\begin{aligned}
\dot{k}_{1} a^{2} & =-2 \frac{r^{2}}{a^{2}}\left(a \frac{d a}{d r} B^{r}+a^{2} \partial_{r} B^{r}\right) \\
\dot{k}_{1} a^{2}+\dot{k}_{2} a^{4} & =-2 \frac{r^{2}}{a^{2}}\left(a \frac{d a}{d r} B^{r}+a^{2} \partial_{z} B^{z}\right) \\
0 & =\partial_{r} B^{z}+\partial_{z} B^{r}
\end{aligned}
$$

From Eq.(33) we find that $B^{r}$ must take the form

$$
B^{r}=\frac{1}{a}\left[F(z, \phi)-\frac{\dot{k}_{1}}{2}\left(-\frac{1}{r}+3 r+r^{3}+\frac{r^{5}}{5}\right)\right]
$$

for some function $F(z, \phi)$. Then using Eq.(36) and integrating Eq.(35) we find

$$
B^{z}=G(z, \phi)-\left(\frac{\partial F}{\partial z}\right) \arctan r
$$

for some function $G(z, \phi)$. Finally, substituting the expressions in equations (36) and (37) into Eq. (34) we find

$$
-\frac{1}{2 r^{2}}\left(\dot{k}_{1} a^{2}+\dot{k}_{2} a^{4}\right)=\frac{\partial G}{\partial z}-\left(\frac{\partial^{2} F}{\partial z^{2}}\right) \arctan r+2 F\left(\frac{r}{a^{2}}\right)-\frac{\dot{k}_{1}}{a^{2}}\left(-1+3 r^{2}+r^{4}+\frac{r^{6}}{5}\right)
$$

The only odd functions of $r$ in this equation are arctan $r$ and $r / a^{2}$ and these functions are linearly independent, so the coefficient of each must vanish. This implies that $F=0$. Furthermore, in order that the left hand side not diverge as $r \rightarrow 0$ we must have $\dot{k}_{2}=-\dot{k}_{1}$. Equation (38) then becomes

$$
\frac{\partial G}{\partial z}=\dot{k}_{1}\left[\frac{a^{2}(1+a)}{2}+\frac{1}{a^{2}}\left(-1+3 r^{2}+r^{4}+\frac{r^{6}}{5}\right)\right]
$$

It then follows that both $\dot{k}_{1}$ and $\partial G / \partial z$ must vanish. Thus, we have found that $C_{\mu \nu}$ and $B^{\mu}$ take the form

$$
\begin{aligned}
C_{\mu \nu} & =c_{1} h_{\mu \nu}+c_{2} \lambda_{\mu} \lambda_{\nu} \\
B^{\mu} & =k_{4}(\phi) \lambda^{\mu}
\end{aligned}
$$


for some function $k_{4}(\phi)$. We now impose the integrability condition Eq.(14) which forces $\dot{k}_{4}$ to vanish. This implies $k_{4}=c_{4}$ and thus $B^{\mu}=c_{4} \lambda^{\mu}$. It then follows from Eq.(16) that $A$ is independent of $\phi$. Equation (17) then becomes

$$
\partial_{\mu} A=c_{1} \partial_{\mu}(r / a)^{-2}
$$

for which the solution is

$$
A(r)=c_{3}+c_{1} \frac{a^{2}}{r^{2}}
$$

Thus the general Killing tensor of the $r z \phi$ surface is

$$
X_{a b}=c_{1} g_{a b}+c_{2} \lambda_{a} \lambda_{b}+c_{3} \eta_{a} \eta_{b}+2 c_{4} \lambda_{(a} \eta_{b)} .
$$

\section{The Melvin metric}

We are now ready to treat the full Melvin metric by adding the $\tau^{a}$ Killing field to the metric of the previous subsection. We have

$$
\begin{gathered}
h_{\mu \nu}=a^{2}\left(r_{\mu} r_{, \nu}+z,_{\mu} z,_{\nu}\right)+\left(r^{2} / a^{2}\right) \phi_{, \mu} \phi_{,_{\nu}} \\
C_{\mu \nu}=k_{1} h_{\mu \nu}+k_{2} \lambda_{\mu} \lambda_{\nu}+k_{3} \eta_{\mu} \eta_{\nu}+2 k_{4} \lambda_{(\mu} \eta_{\nu)}
\end{gathered}
$$

The $\tau^{a}$ Killing vector has $\epsilon=-1$ and $V=a$. Equation (13) for $\dot{C}_{\mu \nu}$ becomes the following:

$$
\begin{aligned}
\dot{k}_{1} & =2 a^{2} \partial_{r} B^{r}+2 a \frac{d a}{d r} B^{r} \\
\dot{k}_{1}+\dot{k}_{2} a^{2} & =2 a \frac{d a}{d r} B^{r}+2 a^{2} \partial_{z} B^{z} \\
a^{-2} \dot{k}_{1}+\frac{r^{2}}{a^{4}} \dot{k}_{3} & =2 \partial_{\phi} B^{\phi}+2\left(\frac{1}{r}-\frac{1}{a} \frac{d a}{d r}\right) B^{r} \\
0 & =\partial_{r} B^{z}+\partial_{z} B^{r} \\
0 & =a^{2} \partial_{\phi} B^{r}+\frac{r^{2}}{a^{2}} \partial_{r} B^{\phi} \\
\dot{k}_{4} & =\frac{a^{4}}{r^{2}} \partial_{\phi} B^{z}+\partial_{z} B^{\phi}
\end{aligned}
$$

Solving Eq.(47) we find

$$
B^{r}=\frac{1}{a}\left[\frac{\dot{k}_{1}}{2} \arctan r+F(z, \phi, t)\right]
$$

for some function $F(z, \phi, t)$. Now, using this result in Eq.(50) we find

$$
B^{z}=-\frac{\partial F}{\partial z} \arctan r+G(z, \phi, t)
$$


for some function $G(z, \phi, t)$. Using equations (53) and (54) in Eq.(48), we obtain

$$
0=-\dot{k}_{1}+\left(2 \frac{\partial G}{\partial z}-\dot{k}_{2}\right) a^{2}+4 F r+2 \dot{k}_{1} r \arctan r-2\left(\frac{\partial^{2} F}{\partial z^{2}}\right) a^{2} \arctan r
$$

Here we have grouped terms so that each term consists of a function of $r$ multiplied by a coefficient that is independent of $r$. Since the functions of $r$ are linearly independent, it follows that each coefficient vanishes. We then find that $F=0$, and that $k_{1}=c_{1}$, and that $G=\dot{k}_{2} z / 2+h(\phi, t)$ for some function $h(\phi, t)$. That is, $B^{r}$ vanishes, and $B^{z}$ takes the form

$$
B^{z}=\frac{1}{2} \dot{k}_{2} z+h(\phi, t)
$$

Equations (49,51,52) then become

$$
\begin{aligned}
\left(\frac{r^{2}}{a^{4}}\right) \dot{k}_{3} & =2 \partial_{\phi} B^{\phi} \\
0 & =\partial_{r} B^{\phi} \\
\dot{k}_{4} & =\left(\frac{a^{4}}{r^{2}}\right) \frac{\partial h}{\partial \phi}+\partial_{z} B^{\phi}
\end{aligned}
$$

Differentiating equations (57) and (59) with respect to $r$, and using Eq.(58), it follows that $\dot{k}_{3}=0$ and $\partial h / \partial \phi=0$. Thus we have $k_{3}=c_{3}$ for constant $c_{3}$, and $h=k_{5}$ for some function $k_{5}(t)$, and $B^{\phi}=\dot{k}_{4} z+k_{6}$ for function $k_{6}(t)$. $B^{\mu}$ takes the form

$$
B^{\mu}=\left(\frac{1}{2} \dot{k}_{2} z+k_{5}\right) \lambda^{\mu}+\left(\dot{k}_{4} z+k_{6}\right) \eta^{\mu}
$$

We therefore have

$$
a^{-2} \dot{B}_{\mu}=\left(\frac{1}{2} \ddot{k}_{2} z+\dot{k}_{5}\right) \partial_{\mu} z+\frac{r^{2}}{a^{4}}\left(\ddot{k}_{4} z+\dot{k}_{6}\right) \partial_{\mu} \phi
$$

Using the expression in Eq.([61), we find that Eq.(14) becomes

$$
0=\ddot{k}_{4} z+\dot{k}_{6}
$$

From Eq.(62) we find that $k_{6}=c_{6}$ and $k_{4}=c_{4} t+c_{7}$ for constants $c_{4}, c_{6}$, and $c_{7}$. Using the expression for $B^{\mu}$ of Eq.(60) in Eq.(15), we find

$$
0=a^{2}\left(\frac{1}{2} \dddot{k}_{2} z+\ddot{k}_{5}\right) \partial_{\mu} z+a^{-1}\left(\frac{d a}{d r}\right) \dot{k}_{1} \partial_{\mu} r
$$

from which it follows that $\dot{k}_{1}$ and $\ddot{k}_{5}$ and $\dddot{k}_{2}$ all vanish. Thus we have $k_{1}=c_{1}$ and $k_{5}=c_{5} t+c_{8}$ and $k_{2}=c_{2} t^{2}+c_{9} t+c_{10}$ for constants $c_{1}, c_{2}, c_{5}, c_{8}, c_{9}$, and $c_{10}$. 
To summarize, we have found that the general solution for $C_{\mu \nu}$ and $B_{\mu}$ takes the form

$$
\begin{aligned}
C_{\mu \nu} & =c_{1} h_{\mu \nu}+\left(c_{2} t^{2}+c_{9} t+c_{10}\right) \lambda_{\mu} \lambda_{\nu}+c_{3} \eta_{\mu} \eta_{\nu}+\left(c_{4} t+c_{7}\right) 2 \lambda_{(\mu} \eta_{\nu)} \\
B_{\mu} & =\left[\left(c_{2} t+\frac{1}{2} c_{9}\right) z+\left(c_{5} t+c_{8}\right)\right] \lambda_{\mu}+\left(c_{4} z+c_{6}\right) \eta_{\mu}
\end{aligned}
$$

It remains to find $A$. Since $B^{r}=0$, it follows from Eq.(16) that $\dot{A}=0$. Using the expressions of equations (64) and (65) in Eq.(17) for grad A, we find

$$
\partial_{\mu} A=2\left(c_{2} z+c_{5}\right) \partial_{\mu} z-c_{1} \partial_{\mu}\left(a^{-2}\right) .
$$

The general solution of Eq.(66) is

$$
A=c_{2} z^{2}+2 c_{5} z-c_{1} a^{-2}+c_{11}
$$

Finally, using the expressions of equations (64-67) in Eq.(4) we find that the most general Killing tensor of Melvin's magnetic universe takes the 4-dimensional form

$$
\begin{aligned}
X_{a b} & =c_{1} g_{a b}+c_{2}\left[z^{2} \tau_{a} \tau_{b}+z t \lambda_{(a} \tau_{b)}+t^{2} \lambda_{a} \lambda_{b}\right]+c_{3} \eta_{a} \eta_{b} \\
& +c_{4}\left[2 z \eta_{(a} \tau_{b)}+2 t \eta_{(a} \lambda_{b)}\right]+c_{5}\left[2 z \tau_{a} \tau_{b}+2 t \lambda_{(a} \tau_{b)}\right]+c_{6} 2 \eta_{(a} \tau_{b)} \\
& +c_{7} 2 \lambda_{(a} \eta_{b)}+c_{8} 2 \lambda_{(a} \tau_{b)}+c_{9}\left[z \lambda_{(a} \tau_{b)}+t \lambda_{a} \lambda_{b}\right]+c_{10} \lambda_{a} \lambda_{b}+c_{11} \tau_{a} \tau_{b}
\end{aligned}
$$

This expression can be simplified by noting that the Melvin boost Killing vector is given by $\psi^{a}:=t \lambda^{a}+z \tau^{a}$. The Killing tensor is then

$$
\begin{aligned}
X_{a b} & =c_{1} g_{a b}+c_{2} \psi_{a} \psi_{b}+c_{3} \eta_{a} \eta_{b}+2 c_{4} \eta_{(a} \psi_{b)}+2 c_{5} \psi_{(a} \tau_{b)}+2 c_{6} \eta_{(a} \tau_{b)} \\
& +2 c_{7} \lambda_{(a} \eta_{b)}+2 c_{8} \lambda_{(a} \tau_{b)}+c_{9} \psi_{(a} \lambda_{b)}+c_{10} \lambda_{a} \lambda_{b}+c_{11} \tau_{a} \tau_{b}
\end{aligned}
$$

Now each term in the sum is either the metric or the symmetrized product of two Killing vectors. Thus all the Killing tensors of Melvin's magnetic universe are trivial.

\section{KILLING TENSORS OF THE SCHWARZSCHILD METRIC}

The Schwarzschild vacuum solution is given by

$$
d s_{\text {Sch }}^{2}=-F d t^{2}+F^{-1} d r^{2}+r^{2}\left(d \vartheta^{2}+\sin ^{2} \vartheta d \varphi^{2}\right) .
$$


where the function $F$ is $F=1-(2 m / r)$. The metric admits four Killing vectors $\left(\tau^{a}, \alpha^{a}, \beta^{a}, \gamma^{a}\right)$; a timelike Killing vector $\tau^{a} \partial_{a}=\partial_{t}$, and three spacelike vectors which comprise the $\mathrm{SO}_{3}$ rotations

$$
\begin{aligned}
\alpha^{a} \partial_{a} & =\sin \varphi \partial_{\vartheta}+\cot \vartheta \cos \varphi \partial_{\varphi} \\
\beta^{a} \partial_{a} & =-\cos \varphi \partial_{\vartheta}+\cot \vartheta \sin \varphi \partial_{\varphi} \\
\gamma^{a} \partial_{a} & =\partial_{\varphi} \\
2 r^{2} & =\alpha^{a} \alpha_{a}+\beta^{a} \beta_{a}+\gamma^{a} \gamma_{a} .
\end{aligned}
$$

Each of the four Killing vectors is hypersurface orthogonal.

First we will find the Killing tensors of the 2-dimensional $r \vartheta$ surface. As before, we will use $c_{1}, c_{2}$ etc. to denote constants, and $k_{1}, k_{2}$ etc. to denote quantities that depend only on the coordinate associated with the Killing vector.

\section{A. An $r \vartheta$ surface}

The $r \vartheta$ 2-surface has metric

$$
g_{a b} d x^{a} d x^{b}=F^{-1} d r^{2}+r^{2} d \vartheta^{2}
$$

We have the $\vartheta$ coordinate Killing vector $\partial_{\vartheta}=\vartheta^{a} \partial_{a}$ for which $\epsilon=1$ and $V=r$ (note that $\vartheta^{a}$ is a symmetry of the 2-surface but not of the spacetime). The metric on the space orthogonal to the Killing vector is $h_{\mu \nu}=F^{-1} r_{, \mu} r,{ }_{\nu}$. Since the r-direction is a 1-dimensional line, it follows as before that the Killing tensor on that 1-dimensional space takes the form

$$
C_{\mu \nu}=k_{1} h_{\mu \nu}=k_{1} F^{-1} r_{, \mu} r_{\nu}
$$

for some $k_{1}(\vartheta)$. Equation (13) then becomes, with $B^{\mu} \rightarrow B^{r}$ only

$$
\dot{C}_{r r}=\dot{k}_{1} F^{-1}=-r^{2}\left[-\left(\frac{2 m}{r^{2}}\right) F^{-2} B^{r}+2 F^{-1} \frac{d B^{r}}{d r}\right]
$$

or

$$
\dot{k}_{1}=2 m F^{-1} B^{r}-2 r^{2} \frac{d B^{r}}{d r}
$$

The general solution for $B^{r}$ is

$$
B^{r}=-\left(\frac{\dot{k}_{1}}{2 m}\right) F+k_{2} F^{1 / 2}
$$


Equation (14) is automatically satisfied, while Eq.(15) becomes

$$
\left(\ddot{k}_{2}+\frac{1}{2} k_{2}\right) F^{-1 / 2}+\left(\frac{\dot{k}_{1}}{m}-\frac{\dddot{k_{1}}}{2 m}\right)+\frac{1}{2} k_{2} F^{1 / 2}-\frac{3}{2 m} \dot{k}_{1} F
$$

Here we have grouped terms so that each term is a coefficient independent of $r$ multiplied by a function of $r$, and the functions of $r$ are linearly independent. Thus each coefficient must vanish, which yields $k_{2}=0$ and $\dot{k}_{1}=0$, from which it follows that $k_{1}=c_{1}$ and $B^{r}=0$. It then follows from Eq.(7) that $A$ is independent of $\vartheta$. From Eq.(17) integration provides

$$
A(r)=\frac{c_{1}}{r^{2}}+c_{2}
$$

Using Eq.(4) we find that the general Killing tensor of the $r \vartheta$ surface is

$$
X_{a b}=c_{1} g_{a b}+c_{2} \vartheta_{a} \vartheta_{b}
$$

\section{B. A $\operatorname{tr} \vartheta$ surface}

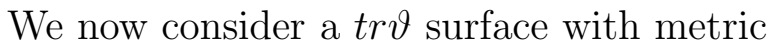

$$
g_{a b} d x^{a} d x^{b}=-F d t^{2}+F^{-1} d r^{2}+r^{2} d \vartheta^{2}
$$

The Killing field is $\tau^{a} \partial_{a}=\partial_{t}$, with $\epsilon=-1$ and $V=F^{1 / 2}$. Here $h_{\mu \nu}$ is the $g_{a b}$ of the previous subsection, while $C_{\mu \nu}$ is the $X_{a b}$ of the previous subsection. We have

$$
\begin{aligned}
h_{\mu \nu} & =F^{-1} r_{, \mu} r_{, \nu}+r^{2} \vartheta_{, \mu} \vartheta_{{ }_{\nu}} \\
C_{\mu \nu} & =k_{1} h_{\mu \nu}+k_{2} \vartheta_{\mu} \vartheta_{\nu} .
\end{aligned}
$$

With $B^{\mu} \rightarrow\left(B^{r}, B^{\vartheta}\right)$, the $r r, \vartheta \vartheta$, and $r \vartheta$ components of Eq.(13) are then, respectively

$$
\begin{aligned}
\dot{k}_{1} F^{-1} & =2 \partial_{r} B^{r}-\frac{2 m}{r^{2}} F^{-1} B^{r} \\
\dot{k}_{1} r^{2}+\dot{k}_{2} r^{4} & =2 r^{2} F \partial_{\vartheta} B^{\vartheta}+2 r F B^{r} \\
0 & =r^{2} \partial_{r} B^{\vartheta}+F^{-1} \partial_{\vartheta} B^{r}
\end{aligned}
$$

From Eq.(82) we find that $B^{r}$ must take the form

$$
B^{r}=F^{1 / 2} H(t, \vartheta)+\frac{1}{2} \dot{k}_{1}\left\{[r-6 m]+3 m F^{1 / 2} \ln \left[\frac{r}{m}\left(1+F^{1 / 2}\right)-1\right]\right\}
$$


for some function $H(t, \vartheta)$. Using the expression of Eq. (85) in Eq. (84) we find

$$
\partial_{r} B^{\vartheta}=-r^{-2} F^{-1 / 2} \partial_{\vartheta} H
$$

Upon integration, it follows that $B^{\vartheta}$ is

$$
B^{\vartheta}=Q(t, \vartheta)-\frac{1}{m} F^{1 / 2} \partial_{\vartheta} H
$$

for integration function $Q(t, \vartheta)$. Substituting the expressions for $B^{r}$ and $B^{\vartheta}$ from equations (85) and (87) into Eq. (83) provides

$$
\begin{aligned}
\dot{k}_{1}+\dot{k}_{2} r^{2} & =\frac{2 F}{r}\left[F^{1 / 2} H(t, \vartheta)+\frac{1}{2} \dot{k}_{1}\left\{[r-6 m]+3 m F^{1 / 2} \ln \left[\frac{r}{m}\left(1+F^{1 / 2}\right)-1\right]\right\}\right] \\
& +2 F\left[\partial_{\vartheta} Q-\frac{1}{m} F^{1 / 2} \frac{\partial^{2} H}{\partial \vartheta^{2}}\right] .
\end{aligned}
$$

Note that each term in Eq. (88) is a function of $r$ multiplied by a coefficient that is independent of $r$. Since the function of $r$ that has the logarithmic term in Eq.(88) is linearly independent of the other functions of $r$, its coefficient must vanish. This implies that $\dot{k}_{1}=0$ and that $k_{1}=c_{1}$ for some constant $c_{1}$. Equation (88) then simplifies to

$$
0=-\frac{1}{2} \dot{k}_{2}\left(\frac{r^{2}}{F}\right)+H\left(\frac{F^{1 / 2}}{r}\right)+\partial_{\vartheta} Q-\frac{1}{m} \frac{\partial^{2} H}{\partial \vartheta^{2}}\left(F^{1 / 2}\right) .
$$

Here terms are grouped so that each term is a coefficient independent of $r$ multiplied by a function of $r$, and so that the functions of $r$ are linearly independent. It therefore follows that each of the coefficients vanishes. We have $\dot{k}_{2}=0, H=0$, and $\partial_{\vartheta} Q=0$. Therefore $k_{2}=c_{2}$ for some constant $c_{2}$, and the components of vector field $B^{\mu}$ are

$$
B^{r}=0, \quad B^{\vartheta}=k_{3}(t)
$$

for some function $k_{3}(t)$. Equivalently

$$
B_{\mu}=k_{3} r^{2} \partial_{\mu} \vartheta
$$

Since $k_{1}=c_{1}$ and $k_{2}=c_{2}$, it follows from Eq.(81) that tensor $C_{\mu \nu}$ takes the form

$$
C_{\mu \nu}=c_{1} h_{\mu \nu}+c_{2} r^{4} \vartheta_{, \mu} \vartheta_{, \nu}
$$

Upon using equations (91) and (92) in Eq.(14) we find that

$$
\partial_{[\mu}\left(F^{-1} r^{2} \dot{k}_{3} \partial_{\nu]} \vartheta\right)=0
$$


from which it follows that $\dot{k}_{3}=0$ and therefore that $k_{3}=c_{3}$ for some constant $c_{3}$. Thus, from Eq.(91) we have

$$
B_{\mu}=c_{3} r^{2} \partial_{\mu} \vartheta
$$

Equation (15) is identically satisfied by equations (92) and (94).

Since $B^{r}=0$ it follows from Eq.(16) that $\dot{A}=0$. Using equations (92) and (94) in Eq.(17) we obtain

$$
\partial_{\mu} A=-c_{1} \partial_{\mu}\left(F^{-1}\right)
$$

It then follows that

$$
A=c_{4}-c_{1} F^{-1}
$$

for some constant $c_{4}$. Finally, using the results of equations (92), (94) and (96) in Eq.(41), we find that the general Killing tensor of a $\operatorname{tr} \theta$ surface is

$$
X_{a b}=c_{1} g_{a b}+c_{2} \vartheta_{a} \vartheta_{b}+2 c_{3} \vartheta_{(a} \tau_{b)}+c_{4} \tau_{a} \tau_{b}
$$

\section{The Schwarzschild metric}

We are now ready to treat the full Schwarzschild metric by adding the axial $\gamma^{a}$ Killing vector to the metric of the previous subsection. We have

$$
\begin{aligned}
& h_{\mu \nu}=-F t_{, \mu} t_{, \nu}+F^{-1} r_{, \mu} r_{, \nu}+r^{2} \vartheta_{, \mu} \vartheta_{, \nu} \\
& C_{\mu \nu}=k_{1} h_{\mu \nu}+k_{2} \vartheta_{\mu} \vartheta_{\nu}+2 k_{3} \vartheta_{(\mu} \tau_{\nu)}+k_{4} \tau_{\mu} \tau_{\nu}
\end{aligned}
$$

The $\gamma^{a}$ Killing vector has $\epsilon=1$ and $V=r \sin \vartheta$. Equation (13) for $\dot{C}_{\mu \nu}$ then becomes

$$
\begin{aligned}
-\dot{k}_{1} F+\dot{k}_{4} F^{2} & =r^{2} \sin ^{2} \vartheta\left(B^{r} \partial_{r} F+2 F \partial_{t} B^{t}\right) \\
\dot{k}_{1} F^{-1} & =r^{2} \sin ^{2} \vartheta\left(F^{-2} B^{r} \partial_{r} F-2 F^{-1} \partial_{r} B^{r}\right) \\
\dot{k}_{1} r^{2}+\dot{k}_{2} r^{4} & =-2 r^{3} \sin ^{2} \vartheta\left(B^{r}+r \partial_{\vartheta} B^{\vartheta}\right) \\
0 & =F^{-1} \partial_{t} B^{r}-F \partial_{r} B^{t} \\
-\dot{k}_{3} r^{2} F & =r^{2} \sin ^{2} \vartheta\left(F \partial_{\vartheta} B^{t}-r^{2} \partial_{t} B^{\vartheta}\right) \\
0 & =r^{2} \partial_{r} B^{\vartheta}+F^{-1} \partial_{\vartheta} B^{r}
\end{aligned}
$$

Equation (101) can be rewritten as

$$
\partial_{r}\left(F^{-1 / 2} B^{r}\right)=-\frac{\dot{k}_{1}}{2 F^{1 / 2} r^{2} \sin ^{2} \vartheta}
$$


with integral

$$
B^{r}=G(t, \vartheta, \varphi) F^{1 / 2}-\frac{\dot{k}_{1}}{2 m \sin ^{2} \vartheta} F
$$

for integration function $G(t, \vartheta, \varphi)$. Using the result of Eq.(107) in Eq.(105), we find

$$
\partial_{r} B^{\vartheta}=-\frac{\partial_{\vartheta} G}{r^{2} F^{1 / 2}}-\frac{\dot{k}_{1} \cos \vartheta}{m r^{2} \sin ^{3} \vartheta} .
$$

Integration provides

$$
B^{\vartheta}=H(t, \vartheta, \varphi)-\left(\partial_{\vartheta} G\right) \frac{1}{m} F^{1 / 2}+\frac{\dot{k}_{1} \cos \vartheta}{m r \sin ^{3} \vartheta}
$$

with integration function $H(t, \vartheta, \varphi)$. Now using equations (107) and (109) in Eq.(102) we obtain

$0=-3 \dot{k}_{1}-\left(\dot{k}_{2}+\partial_{\vartheta} H 2 \sin ^{2} \vartheta\right) r^{2}+\frac{\dot{k}_{1}}{m}\left(\frac{6}{\sin ^{2} \vartheta}-3\right) r-2 \sin ^{2} \vartheta G\left(r F^{1 / 2}\right)+\frac{2}{m} \sin ^{2} \vartheta\left(\frac{\partial^{2} G}{\partial \vartheta^{2}}\right) r^{2} F^{1 / 2}$

Here we have grouped terms so that each term is a coefficient independent of $r$ multiplied by a function of $r$, and so that the functions of $r$ are linearly independent. It therefore follows that each coefficient vanishes. Thus $G=\dot{k}_{1}=0$ and

$$
\partial_{\vartheta} H=-\frac{\dot{k}_{2}}{2 \sin ^{2} \vartheta} \text {. }
$$

From the vanishing of $G$ and $\dot{k}_{1}$ it follows that $B^{r}$ vanishes, and that $k_{1}=c_{1}$ for some constant $c_{1}$ and that $B^{\vartheta}=H$. From Eq.(111) it follows that $B^{\vartheta}$ takes the form

$$
B^{\vartheta}=I(t, \varphi)+\frac{\dot{k}_{2}}{2} \cot \vartheta
$$

for some function $I(t, \varphi)$. Using Eq.(112), along with $B^{r}=0$ and $\dot{k}_{1}=0$, reduces equations (100, 105) to the following:

$$
\begin{aligned}
\partial_{t} B^{t} & =\frac{\dot{k}_{4} F}{2 r^{2} \sin ^{2} \vartheta} \\
\partial_{r} B^{t} & =0 \\
\partial_{\vartheta} B^{t} & =r^{2} F^{-1} \partial_{t} I-\frac{\dot{k}_{3}}{\sin ^{2} \vartheta}
\end{aligned}
$$

Applying $\partial_{r}$ to Eq.(113) and using Eq.(114) yields $\dot{k}_{4}=0$. Therefore $B^{t}$ is independent of $t$ and $k_{4}=c_{2}$ for some constant $c_{2}$. Now applying $\partial_{r}$ to Eq.(115) and using Eq.(114) it follows that $\partial_{t} I=0$ and $I=k_{5}$ for some function $k_{5}(\varphi)$. Thus $B^{\vartheta}$ becomes

$$
B^{\vartheta}=k_{5}+\frac{\dot{k}_{2}}{2} \cot \vartheta
$$


Integrating Eq.(115) yields

$$
B^{t}=k_{6}+\dot{k}_{3} \cot \vartheta
$$

for some function $k_{6}(\varphi)$. In summary, we have found that $B_{\mu}$ and $C_{\mu \nu}$ take the form

$$
\begin{aligned}
B_{\mu} & =\left(k_{6}+\dot{k}_{3} \cot \vartheta\right) \tau_{\mu}+\left(k_{5}+\frac{\dot{k}_{2}}{2} \cot \vartheta\right) \vartheta_{\mu} \\
C_{\mu \nu} & =c_{1} h_{\mu \nu}+k_{2} \vartheta_{\mu} \vartheta_{\nu}+2 k_{3} \vartheta_{(\mu} \tau_{\nu)}+c_{2} \tau_{\mu} \tau_{\nu}
\end{aligned}
$$

We now impose the integrability conditions of equations (14) and (15) on the expressions above for $B_{\mu}$ and $C_{\mu \nu}$. From equations (118) and (119), with $\epsilon=1$ and $V=r \sin \vartheta$, we find

$$
\begin{aligned}
V^{-2} \dot{B}_{\mu}+V^{-3} C_{\mu \nu} \partial^{\nu} V & =c_{1} V^{-3} \partial_{\mu} V-\frac{F}{r^{2} \sin ^{2} \vartheta}\left[\dot{k}_{6}+\left(\ddot{k}_{3}+k_{3}\right) \cot \vartheta\right] \partial_{\mu} t \\
& +\frac{1}{\sin ^{2} \vartheta}\left[\dot{k}_{5}+\left(\frac{\ddot{k}_{2}}{2}+k_{2}\right) \cot \vartheta\right] \partial_{\mu} \vartheta
\end{aligned}
$$

The integrability condition given in Eq.(14) is the statement that the right hand side of Eq.(120) is curl-free. From this it follows that, for the term in Eq.(120) multiplying $\partial_{\mu} t$, the quantity in square brackets vanishes. That is, we have $\dot{k}_{6}=0$ and $\ddot{k}_{3}+k_{3}=0$. Thus

$$
\begin{aligned}
& k_{6}=c_{3} \\
& k_{3}=c_{4} \cos \varphi+c_{5} \sin \varphi
\end{aligned}
$$

for constants $c_{3}, c_{4}$, and $c_{5}$.

From equations (118), (120), (121), and (122) it follows that

$$
\ddot{B}_{\mu}+V^{-1} \dot{C}_{\mu \nu} \partial^{\nu} V-\epsilon V^{2} \partial_{\mu}\left(V^{-1} B^{\nu} \partial_{\nu} V\right)=\left[\ddot{k}_{5}+k_{5}+\left(\frac{\dddot{k}_{2}}{2}+2 \dot{k}_{2}\right) \cot \vartheta\right] r^{2} \partial_{\mu} \vartheta
$$

The integrability condition of Eq.(15) states that the right hand side of Eq.(123) vanishes. Therefore $\ddot{k}_{5}+k_{5}=0$ and $\dddot{k}_{2}+4 \dot{k}_{2}=0$, and thus

$$
\begin{aligned}
& k_{5}=c_{6} \cos \varphi+c_{7} \sin \varphi \\
& k_{2}=c_{8} \cos 2 \varphi+c_{9} \sin 2 \varphi+c_{10}
\end{aligned}
$$

for constants $c_{6}, c_{7}, c_{8}, c_{9}$, and $c_{10}$. We find that the general solution for $B_{\mu}$ and $C_{\mu \nu}$ is

$$
\begin{aligned}
B_{\mu} & =\left[c_{3}+\left(-c_{4} \sin \varphi+c_{5} \cos \varphi\right) \cot \vartheta\right] \tau_{\mu} \\
& +\left[c_{6} \cos \varphi+c_{7} \sin \varphi+\left(-c_{8} \sin 2 \varphi+c_{9} \cos 2 \varphi\right) \cot \vartheta\right] \vartheta_{\mu} \\
C_{\mu \nu} & =c_{1} h_{\mu \nu}+\left(c_{8} \cos 2 \varphi+c_{9} \sin 2 \varphi+c_{10}\right) \vartheta_{\mu} \vartheta_{\nu} \\
& +2\left(c_{4} \cos \varphi+c_{5} \sin \varphi\right) \vartheta_{(\mu} \tau_{\nu)}+c_{2} \tau_{\mu} \tau_{\nu}
\end{aligned}
$$


It remains to find $A$. Using equations (126) and (127) in Eq.(17) we obtain

$$
\begin{aligned}
\partial_{\mu} A & =-2 c_{1} V^{-3} \partial_{\mu} V \\
& -\frac{2}{\sin ^{2} \vartheta}\left[-c_{6} \sin \varphi+c_{7} \cos \varphi+\left(-c_{8} \cos 2 \varphi-c_{9} \sin 2 \varphi+c_{10}\right) \cot \vartheta\right] \partial_{\mu} \vartheta
\end{aligned}
$$

with general solution

$$
A=c_{1} V^{-2}+2\left(-c_{6} \sin \varphi+c_{7} \cos \varphi\right) \cot \vartheta+\left(-c_{8} \cos 2 \varphi-c_{9} \sin 2 \varphi+c_{10}\right) \cot ^{2} \vartheta+k_{7}
$$

for some function $k_{7}(\varphi)$. Imposing Eq.(16) on Eq.(129) we find that $\dot{k}_{7}=0$ and therefore that $k_{7}=c_{11}$ for some constant $c_{11}$. The solution for $A$ becomes

$$
A=c_{1} V^{-2}+2\left(-c_{6} \sin \varphi+c_{7} \cos \varphi\right) \cot \vartheta+\left(-c_{8} \cos 2 \varphi-c_{9} \sin 2 \varphi+c_{10}\right) \cot ^{2} \vartheta+c_{11}
$$

Using equations (126), (127), and (130) in Eq.(44), and grouping terms according to their constant coefficient, we find that the general Schwarzschild Killing tensor is

$$
\begin{aligned}
X_{a b} & =c_{1}\left(h_{a b}+V^{-2} \gamma_{a} \gamma_{b}\right)+c_{2} \tau_{a} \tau_{b}+c_{3} 2 \tau_{(a} \gamma_{b)} \\
& +c_{4}\left[2 \cos \varphi \vartheta_{(a} \tau_{b)}-2 \sin \varphi \cot \vartheta \gamma_{(a} \tau_{b}\right] \\
& +c_{5}\left[2 \sin \varphi \vartheta_{(a} \tau_{b)}+2 \cos \varphi \cot \vartheta \gamma_{(a} \tau_{b)}\right]+c_{6}\left[2 \cos \varphi \vartheta_{(a} \gamma_{b)}-2 \sin \varphi \cot \vartheta \gamma_{a} \gamma_{b}\right] \\
& +c_{7}\left[2 \sin \varphi \vartheta_{(a} \gamma_{b)}+2 \cos \varphi \cot \vartheta \gamma_{a} \gamma_{b}\right] \\
& +c_{8}\left[\cos 2 \varphi \vartheta_{a} \vartheta_{b}-2 \sin 2 \varphi \cot \vartheta \vartheta_{(a} \gamma_{b)}-\cos 2 \varphi \cot ^{2} \vartheta \gamma_{a} \gamma_{b}\right] \\
& +c_{9}\left[\sin 2 \varphi \vartheta_{a} \vartheta_{b}+2 \cos 2 \varphi \cot \vartheta \vartheta_{(a} \gamma_{b)}-\sin 2 \varphi \cot ^{2} \vartheta \gamma_{a} \gamma_{b}\right] \\
& +c_{10}\left[\vartheta_{a} \vartheta_{b}+\cot ^{2} \vartheta \gamma_{a} \gamma_{b}\right]+c_{11} \gamma_{a} \gamma_{b}
\end{aligned}
$$

We now rewrite this expression for the Killing tensor in terms of Killing vectors and the metric. We have

$$
\begin{aligned}
g_{a b} & =h_{a b}+V^{-2} \gamma_{a} \gamma_{b}, \\
\alpha_{a} & =\sin \varphi \vartheta_{a}+(\cot \vartheta \cos \varphi) \gamma_{a}, \\
\beta_{a} & =-\cos \varphi \vartheta_{a}+(\cot \vartheta \sin \varphi) \gamma_{a},
\end{aligned}
$$

which yields

$$
\begin{aligned}
\alpha_{a} \alpha_{b}+\beta_{a} \beta_{b} & =\vartheta_{a} \vartheta_{b}+\cot ^{2} \vartheta \gamma_{a} \gamma_{b} \\
\alpha_{a} \alpha_{b}-\beta_{a} \beta_{b} & =\cos 2 \varphi\left(-\vartheta_{a} \vartheta_{b}+\cot ^{2} \vartheta \gamma_{a} \gamma_{b}\right)+2 \cot \vartheta \sin 2 \varphi \vartheta_{(a} \gamma_{b)} \\
2 \alpha_{(a} \beta_{b)} & =\sin 2 \varphi\left(-\vartheta_{a} \vartheta_{b}+\cot ^{2} \vartheta \gamma_{a} \gamma_{b}\right)-2 \cot \vartheta \cos 2 \varphi \vartheta_{(a} \gamma_{b)}
\end{aligned}
$$


Using the three equations above, we can rewrite $X_{a b}$ as

$$
\begin{aligned}
X_{a b} & =c_{1} g_{a b}+c_{2} \tau_{a} \tau_{b}+c_{3} 2 \tau_{(a} \gamma_{b)}-2 c_{4} \beta_{(a} \tau_{b)}+2 c_{5} \alpha_{(a} \tau_{b)}-2 c_{6} \beta_{(a} \gamma_{b)}+2 c_{7} \alpha_{(a} \gamma_{b)} \\
& -c_{8}\left(\alpha_{a} \alpha_{b}-\beta_{a} \beta_{b}\right)-2 c_{9} \alpha_{(a} \beta_{b)}+c_{10}\left(\alpha_{a} \alpha_{b}+\beta_{a} \beta_{b}\right)+c_{11} \gamma_{a} \gamma_{b} .
\end{aligned}
$$

Finally, regrouping terms we have

$$
\begin{aligned}
X_{a b} & =c_{1} g_{a b}+c_{2} \tau_{a} \tau_{b}+c_{3} 2 \tau_{(a} \gamma_{b)}-2 c_{4} \beta_{(a} \tau_{b)}+2 c_{5} \alpha_{(a} \tau_{b)}-2 c_{6} \beta_{(a} \gamma_{b)}+2 c_{7} \alpha_{(a} \gamma_{b)} \\
& +\left(c_{10}-c_{8}\right) \alpha_{a} \alpha_{b}+\left(c_{10}+c_{8}\right) \beta_{a} \beta_{b}-2 c_{9} \alpha_{(a} \beta_{b)}+c_{11} \gamma_{a} \gamma_{b} .
\end{aligned}
$$

Thus, we have written the general Schwarzschild Killing tensor as a sum of terms where each term is either the metric or a product of Killing vectors. Therefore all Killing tensors of the Schwarzschild spacetime are trivial.

\section{CONCLUSIONS}

The method used above consists of applying equations (13-17) to find the Killing tensor in $\mathrm{n}$-dimensions, using the equations in $\mathrm{n}-1$ dimensions. For the Melvin metric and the Schwarzschild metric this is done three times, going from a 1-dimensional space to the 4dimensional spacetime.

Professor G. Valent has noted that his recent paper [6] uses a similar method for studying geodesic flows. His method differs from ours. Valent assumes the Killing tensor is Lie derived by the Killing field. We make no such assumption. Here, we assume the Killing field is hypersurface orthogonal.

The method developed here for finding Killing tensors could be used on a wide variety of spacetimes where there are symmetries. It requires less computation than an attack on the full Killing tensor equations, and it can be used even when the spacetime is not algebraically special. The method could also be generalized in various ways. The equations for a Killing-Yano tensor 7] could be treated in an analogous way and should result in a method simpler than a straightforward attempt to solve the Killing-Yano equations. Finally the method might have a useful generalization to the case where the Killing vector is not hypersurface orthogonal. In that case one would expect to get more complicated equations that involve not only the norm of the Killing field but also the twist. However, it is in just 
such spacetimes (i.e. Kerr) that known examples of nontrivial Killing tensors exist. So an investigation along those lines might be useful.

[1] B. Carter, Hamilton-Jacobi and Schrödinger separable solutions of Einstein's equations, Commun. Math. Phys. 10, 280 (1968).

[2] P. Sommers, On Killing tensors and constants of motion, J. Math. Phys. 14, 787 (1973).

[3] Exact Solutions of Einstein's Field Equations, Eds. D. Kramer, H. Stephani, E. Herlt, M. MacCallum and E. Schmutzer, 2nd Ed. (Cambridge University Press, Cambridge, U.K. 2003).

[4] R. Geroch, J. Math. Phys. A Method for Generating New Solutions of Einstein's Equation. II, 13, 394 (1972).

[5] M.A. Melvin, Pure Magnetic and Electric Geons, Phys. Lett. 8, 65 (1964).

[6] G. Valent, Integrability versus separability for the Multi-Centre metrics, Commun. Math. Phys. 244, 571 (2004).

[7] C.D. Collinson, On the Relationship between Killing Tensors and Killing-Yano Tensors, Int. Jour. Theor. Phys. 15, 311 (1976). 